\title{
FACTORIZATIONS IN FINITE GROUPS
}

\author{
VIK.S. KULIKOV
}

\begin{abstract}
A necessary condition for uniqueness of factorizations of elements of a finite group $G$ with factors belonging to a union of some conjugacy classes of $G$ is given. This condition is sufficient if the number of factors belonging to each conjugacy class is big enough. The result is applied to the problem on the number of irreducible components of the Hurwitz space of degree $d$ marked coverings of $\mathbb{P}^{1}$ with given Galois group $G$ and fixed collection of local monodromies.
\end{abstract}

\section{INTRODUCTION}

Let $f: X \rightarrow \mathbb{P}^{1}$ be a morphism of a non-singular irreducible projective curve $X$ (defined over the field of complex numbers $\mathbb{C}$ ) onto the projective line $\mathbb{P}^{1}$. Denote by $\mathbb{C}(X)$ the field of rational functions on $X$. The morphism $f$ defines a finite extension $f^{*}: \mathbb{C}(z) \hookrightarrow \mathbb{C}(X)$ of the field of rational functions $\mathbb{C}\left(\mathbb{P}^{1}\right) \simeq \mathbb{C}(z)$. Denote by $G$ the Galois group of this extension.

Let us choose a point $z_{0} \in \mathbb{P}^{1}$ such that $z_{0}$ is not a branch point of $f$ and number the points of $f^{-1}\left(z_{0}\right)=\left\{w_{1}, \ldots, w_{d}\right\}$, where $d=\operatorname{deg} f$. We will call the morphism $f$ with fixed numbering of the points of $f^{-1}\left(z_{0}\right)$ a marked covering.

Let $z_{1}, \ldots, z_{n} \in \mathbb{P}^{1}$ be the set of branch points of $f$. The numbering of the points of $f^{-1}\left(z_{0}\right)$ defines a homomorphism $f_{*}: \pi_{1}\left(\mathbb{P}^{1} \backslash\left\{z_{1}, \ldots, z_{n}\right\}, z_{0}\right) \rightarrow \Sigma_{d}$ of the fundamental group $\pi_{1}=\pi_{1}\left(\mathbb{P}^{1} \backslash\left\{z_{1}, \ldots, z_{n}\right\}, z_{0}\right)$ to the symmetric group $\Sigma_{d}$. The image $\operatorname{im} f_{*}$ acts transitively on $f^{-1}\left(z_{0}\right)$ and it is isomorphic to $G$ (so we can identify $\operatorname{im} f_{*}$ and $G$ ). Let $\gamma_{1}, \ldots, \gamma_{n}$ be simple loops around, respectively, the points $z_{1}, \ldots, z_{n}$ starting at $z_{0}$ and such that they generate the group $\pi_{1}$. The image $g_{j}=f_{*}\left(\gamma_{j}\right) \in G$ is called a local monodromy of $f$ at the point $z_{j}$. Note that the set $\left\{g_{1}, \ldots, g_{n}\right\}$ of local monodromies generate the group $G$. The local monodromy $g_{j}$ depends on the choice of $\gamma_{j}$, therefore it is defined uniquely up to conjugation in $G$. Denote by $O=C_{1} \sqcup \cdots \sqcup C_{m} \subset G$ the union of conjugacy classes of all local monodromies and by $\tau_{i}$ the number of local monodromies of $f$ belonging to the conjugacy class $C_{i}$. The pair $(G, O)$ is called an equipped group and the collection $\tau=\left(\tau_{1} C_{1}, \ldots, \tau_{m} C_{m}\right)$ is called the monodromy type of $f$.

Let $\operatorname{HUR}_{d, G, \tau}^{m}\left(\mathbb{P}^{1}\right)$ be the Hurwitz space (see the definition of Hurwitz spaces in [1) of marked degree $d$ coverings of $\mathbb{P}^{1}$ with Galois group $G$ and monodromy type $\tau$. The famous Clebsch - Hurwitz Theorem ([2], [3]) states that if $G=\Sigma_{d}$ and $O$ is the

This research was partially supported by grants of NSh-4713.2010.1, RFBR 11-01-00185, and by AG Laboratory HSE, RF government grant, ag. 11.G34.31.0023. 
set of transpositions, then $\operatorname{HUR}_{d, \Sigma_{d}, \tau}^{m}\left(\mathbb{P}^{1}\right)$ consists of a single irreducible component if $\tau=(n O)$ with even $n \geqslant 2(d-1)$ and it is empty overwise. Generalizations of Clebsch - Hurwitz Theorem were obtained in [4] and [5]. In particular, in [5] it was proved that for an equipped group $\left(\Sigma_{d}, O\right)$ with $O=C_{1} \sqcup \cdots \sqcup C_{m}$, where $C_{1}$ is the conjugacy class of an odd permutation leaving fixed at least two elements, the Hurwitz space $\operatorname{HUR}_{d, \Sigma_{d}, \tau}^{m}\left(\mathbb{P}^{1}\right)$ is irreducible if $\tau_{1}$ is big enough. On the other hand, the example in [6] shows that $\operatorname{HUR}_{8, \Sigma_{8}, \tau}^{m}\left(\mathbb{P}^{1}\right)$ consists at least of two irreducible components, where $\tau=\left(1 C_{1}, 1 C_{2}, 1 C_{3}\right)$ and $C_{1}$ is the conjugacy class of permutation $(1,2)(3,4,5), C_{2}$ is the conjugacy class of $(1,2,3)(4,5,6,7)$, and $C_{3}$ is the conjugacy class of $(1,2,3,4,5,6,7)$. Therefore we can not wait that for a fixed equipped finite group $(G, O)$ the number of irreducible components of $\operatorname{HUR}_{d, G, \tau}^{m}\left(\mathbb{P}^{1}\right)$ does not depend on the monodromy type $\tau$. But, we can wait that this number does not depend on $\tau$ if $\tau_{i}$ is big enough for some $i$ such that the elements of $C_{i}$ generate the group $G$.

In subsection 2.5, for each equipped finite group $(G, O)$ such that the elements of $O$ generate $G$, we define a number $a_{(G, O)}$ depending on $G$ and $O$, call it the ambiguity index of $(G, O)$, and, as a straightforward corollary of Theorems 6 and 7 (see subsection 4.2) and results of [4], we have

Theorem 1. For each equipped finite group $(G, O), O=C_{1} \sqcup \cdots \sqcup C_{m}$, such that the elements of $O$ generate the group $G$, there is a constant $T$ such that the number of irreducible components of each non-empty Hurwitz space $H U R_{d, G, \tau}^{m}\left(\mathbb{P}^{1}\right)$ is equal to $a_{(G, O)}$ if $\tau_{i} \geqslant T$ for all $i=1, \ldots, m$.

If the elements of $O_{1}=C_{1} \sqcup \cdots \sqcup C_{k}$ for some $k<m$ generate the group $G$, then there is a constant $T_{1}$ such that the number of irreducible components of $H U R_{d, G, \tau}^{m}\left(\mathbb{P}^{1}\right)$ is not more than $a_{\left(G, O_{1}\right)}$ if $\tau_{i} \geqslant T_{1}$ for $i=1, \ldots, k$.

This article is a continuation of [4], in which the investigation of the factorization semigroups over finite groups was started. For a convenience of the reader, the main definitions and useful statements from [4] are reminded in section 1. In section 2, to each equipped group $(G, O)$, we associate a $C$-group whose factorization semigroup is the same as the factorization semigroup of $(G, O)$ and we investigate a connection between this $C$-group and $(G, O)$. In section 3 , we prove the stability of the factorization semigroups over finite $C$-groups. Theorem 2, proved in this section, plays the key role in the proof of all main results of this article (see section 4). In section 5 , we give a solution of the word problem for finite $C$-groups and give an algorithm of computation of the ambiguity index $a_{(G, O)}$ for an equipped finite group $(G, O)$.

The author is grateful to O.V. Kulikova who draws attention of the author on the important role of van Kampen Lemma in geometric group theory, only due to the Lemma it was possible to prove Theorem 2. 


\section{Semigroups over Groups}

1.1. Factorization semigroups. A pair $(G, O)$, where $G$ is a group and $O$ is a subset of $G$ invariant under the inner automorphisms, is called an equipped group. In the sequel, we will assume that $\mathbf{1} \notin O$ and $O$ consists of a finite number of conjugacy classes $C_{i}$ of $G, O=C_{1} \sqcup \cdots \sqcup C_{m}$, and the numbering of these conjugacy classes is fixed.

A homomorphism $f: G_{1} \rightarrow G_{2}$ is a homomorphism of equipped groups $\left(G_{1}, O_{1}\right)$ and $\left(G_{2}, O_{2}\right)$ if $f\left(O_{1}\right) \subset O_{2}$.

A semigroup $S(G, O)$, generated by the letters of the alphabet $X=X_{O}=\left\{x_{g} \mid\right.$ $g \in O\}$ being subject to the relations

$$
x_{g_{1}} \cdot x_{g_{2}}=x_{g_{2}} \cdot x_{g_{2}^{-1} g_{1} g_{2}}=x_{g_{1} g_{2} g_{1}^{-1}} \cdot x_{g_{1}},
$$

is called the factorization semigroup with factors in $O$ (or a factorization semigroup over the group $G)$. A homomorphism $\alpha=\alpha_{G}: S(G, O) \rightarrow G$, given by $\alpha\left(x_{g}\right)=g$ for each $x_{g} \in X$, is called the product homomorphism. We will denote by $g_{1} \ldots g_{n}$ the image $\alpha\left(x_{g_{1}} \cdot \ldots \cdot x_{g_{n}}\right)$ of $s=x_{g_{1}} \cdot \ldots \cdot x_{g_{n}} \in S(G, O)$.

The action $\rho$ of the group $G$ on the set $X$, given by

$$
x_{a} \in X \mapsto \rho(g)\left(x_{a}\right)=x_{g a g^{-1}} \in X,
$$

defines a homomorphism $\rho: G \rightarrow \operatorname{Aut}(S(G, O))$. The action $\rho(g)$ on $S(G, O)$ is called the simultaneous conjugation by $g \in G$. Put $\lambda(g)=\rho\left(g^{-1}\right)$ and $\lambda_{S}=\lambda \circ \alpha$, $\rho_{S}=\rho \circ \alpha$.

Claim 1. ([4]) For all $s_{1}, s_{2} \in S(G, O)$ we have

$$
s_{1} \cdot s_{2}=s_{2} \cdot \lambda_{S}\left(s_{2}\right)\left(s_{1}\right)=\rho_{S}\left(s_{1}\right)\left(s_{2}\right) \cdot s_{1} \text {. }
$$

To each element $s=x_{g_{1}} \cdot \ldots \cdot x_{g_{n}} \in S(G, O)$, let us associate a positive integer $\ln (s)=n$ called the length of $s$. It is easy to see that $\ln : S(G, O) \rightarrow \mathbb{Z}_{\geqslant 0}=\{\mathbf{a} \in \mathbb{Z} \mid$ $\mathbf{a} \geqslant 0\}$ is a homomorphism of semigroups.

For each $s=x_{g_{1}} \cdot \ldots \cdot x_{g_{n}} \in S(G, O)$ denote by $G_{s}$ the subgroup of $G$ generated by the images $\alpha\left(x_{g_{1}}\right)=g_{1}, \ldots, \alpha\left(x_{g_{n}}\right)=g_{n}$ of the factors $x_{g_{1}}, \ldots, x_{g_{n}}$, and denote by $G_{O}$ the subgroup of $G$ generated by the elements of $O$.

Claim 2. ([4]) The subgroup $G_{s}$ of $G$ is well defined, that is, it does not depend on a presentation of $s$ as a product of generators $x_{g_{i}} \in X_{O}$.

For subgroups $H$ and $Z$ of a group $G$ denote by

$$
\begin{aligned}
& S(G, O)^{H}=\left\{s \in S(G, O) \mid G_{s}=H\right\}, \\
& S(G, O)_{Z}=\{s \in S(G, O) \mid \alpha(s) \in Z\},
\end{aligned}
$$

and $S(G, O)_{Z}^{H}=S(G, O)_{Z} \cap S(G, O)^{H}$. It is easy to see that $S(G, O)^{H}$ (respectively $\left.S(G, O)_{Z}^{H}\right)$ is isomorphic to the semigroup $S(H, H \cap O)^{H}$ (respectively, isomorphic to $\left.S(H, H \cap O)_{Z}^{H}\right)$ and the isomorphism is induced by imbedding $(H, H \cap O) \hookrightarrow(G, O)$.

Proposition 1. ([4]) Let $(G, O)$ be an equipped group and let $s \in S(G, O)$. We have 
(1) ker $\rho$ coincides with the centralizer $C_{O}$ of the group $G_{O}$ in $G$;

(2) if $\alpha(s)$ belongs to the center $Z\left(G_{s}\right)$ of $G_{s}$, then for each $g \in G_{s}$ the action $\rho(g)$ leaves fixed the element $s \in S(G, O)$;

(3) if $\alpha\left(s \cdot x_{g}\right)$ belongs to the center $Z\left(G_{s \cdot x_{g}}\right)$ of $G_{s \cdot x_{g}}$, then $s \cdot x_{g}=x_{g} \cdot s$,

(4) if $\alpha(s)=\mathbf{1}$, then $s \cdot s^{\prime}=s^{\prime} \cdot s$ for any $s^{\prime} \in S(G, O)$.

Claim 3. ([4]) For any equipped group $(G, O)$ the semigroup $S(G, O)_{1}$ is contained in the center of the semigroup $S(G, O)$ and, in particular, it is a commutative subsemigroup.

It is easy to see that if $g \in O$ is an element of order $n$, then $x_{g}^{n} \in S(G, O)_{\mathbf{1}}$.

Lemma 1. ([4]) Let $s \in S(G, O)_{Z\left(G_{O}\right)}$ and $s_{1} \in S(G, O)$ be such that $G_{s_{1}}=G_{O}$, where $Z\left(G_{O}\right)$ is the center of $G_{O}$. Then

$$
s \cdot s_{1}=\rho(g)(s) \cdot s_{1}
$$

for all $g \in G_{O}$.

In particular, if $s \in S(G, O)$ is such that $G_{s}=G$ and $C \subset O$ is a conjugacy class of $G$, then for any $g_{1}, g_{2} \in C$ we have

$$
x_{g_{1}}^{n} \cdot s=x_{g_{2}}^{n} \cdot s
$$

if $g_{1}^{n}$ belongs to the center $Z(G)$ of $G$.

Proposition 2. ([4]) The elements of $S(G, O)_{1}^{G}$ are fixed under the conjugation action of $G$.

\section{2. $C$-groups AND $C$-GRAPHS}

2.1. $C$-graphs of $C$-groups. Remind the definition of $C$-groups. By definition (see, for example, [7]), a $C$-group $G$ is an equipped group $(G, O)$ such that the elements of $O$ are the generators (so called, $C$-generators) of the group $G$ being subject to the relations

$$
g_{i}^{-1} g_{j} g_{i}=g_{k}, \quad\left(g_{i}, g_{j}, g_{k}\right) \in M
$$

where $M$ is a subset of $O^{3}$. A homomorphism $f: G_{1} \rightarrow G_{2}$ of $C$-groups is called a $C$-homomorphism if it is a homomorphism of equipped groups. In particular, two $C$-groups $G_{1}$ and $G_{2}$ are $C$-isomorphic if they are isomorphic as equipped groups.

To each $C$-group, we can associate a directed graph, called $C$-graph. To give a definition of $C$-graphs, consider a directed graph $\Gamma=(V, E)$, where $V$ is the set of vertices of $\Gamma$ and the set of its edges $E$ is a collection $\left\{e_{v_{i}, v_{j}}=\left(v_{i}, v_{j}\right)\right\}$ of ordered pairs of its vertices (some of edges can be loops, that is, the equality $v_{i}=v_{j}$ is allowed). For each vertex $v \in V$, let us denote by $T_{v}=\left\{e_{v, v_{i}}\right\}$ (resp., $H_{v}=\left\{e_{v_{i}, v}\right\}$ ) the set of edges whose tails (resp., heads) are the vertex $v$. A directed graph $\Gamma$ is called a $C$-graph if each edge $e \in E$ is labeled by an element of $V$, that is, there is a map $f: E \rightarrow V$ so that the label of $e \in E$ is $f(e) \in V$ (in the sequel, an edge $e_{v_{1}, v_{2}}$ with label $f\left(e_{v_{1}, v_{2}}\right)=v$ will be denoted by $\left.e_{v_{1}, v_{2}, v}\right)$, and $\Gamma$ is such that the following five conditions are satisfied: 
(i) for each vertex $v \in V$ the restrictions $f_{\mid T_{v}}, f_{\mid H_{v}}$ of the map $f$ to $T_{v}$ and $H_{v}$ are one to one correspondences with $V$;

(ii) for each vertex $v$ the head $v_{1}$ of the edge $e_{v, v_{1}, v}$ is the vertex $v$, that is, $e_{v, v_{1}, v}$ is the loop $\left(v_{1}=v\right)$;

Note that, by condition $(i)$, the tail (resp., the head) $v_{1}$ and the label $v_{2}$ define uniquely the edge $e$ whose tail (resp., head) is $v_{1}$ and whose label is $v_{2}$. Therefore a sequence $v_{1}, \ldots, v_{n}$ and a tail $v_{0}$ define uniquely a path $l\left(v_{0} ; v_{1}, \ldots, v_{n}\right)$ starting at the vertex $v_{0}$ along edges (in the positive direction) with labels $v_{1}, \ldots, v_{n}$.

The third and fourth conditions are

(iii) if for some two vertices $v_{1}$ and $v_{2}$ the edge $e_{v_{1}, v_{3}, v_{2}}$ is a loop, that is, $v_{1}=v_{3}$, then the edge $e_{v_{2}, v_{4}, v_{1}}$ is also a loop $\left(v_{2}=v_{4}\right)$.

(iv) for any edge $e_{v_{1}, v_{2}, v_{3}}$ and for any vertex $v$ the ends of the paths $l\left(v ; v_{1}, v_{3}\right)$ and $l\left(v ; v_{3}, v_{2}\right)$ coincide.

To each labeled directed graph $\Gamma$, let us associate a two-dimensional complex $K_{\Gamma}$

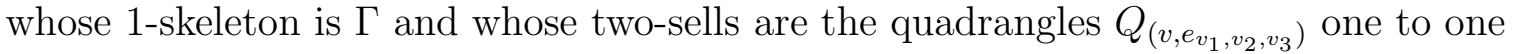
corresponding to the pairs $\left(v, e_{v_{1}, v_{2}, v_{3}}\right) \in V \times E$ such that the border $\partial Q_{\left(v, e_{v_{1}, v_{2}, v_{3}}\right)}$ of $Q_{\left(v, e_{\left.v_{1}, v_{2}, v_{3}\right)}\right.}$ is the loop $l\left(v ; v_{1}, v_{3}\right) \cdot l\left(v ; v_{3}, v_{2}\right)^{-1}$.

The fifth condition is

$(v)$ if for some two edges $e_{v, v_{1}, v_{2}}$ and $e_{v, v_{1}, v_{3}}$ the loop $e_{v, v_{1}, v_{2}} \cdot e_{v, v_{1}, v_{3}}^{-1}$ represents the unity of the fundamental group $\pi_{1}\left(K_{\Gamma}, v\right)$, then $v_{2}=v_{3}$.

To each $C$-group $(G, O)$, one can associate a $C$-graph. By definition, the $C$-graph $\Gamma=\Gamma_{(G, O)}$ of a $C$-group $(G, O)$ is a $C$-graph whose set of vertices $V=\left\{v_{g_{i}} \mid g_{i} \in O\right\}$ is in one to one correspondence with the set $O$. Two vertices $v_{g_{1}}$ and $v_{g_{2}}, g_{1}, g_{2} \in O$ are connected by a labeled edge $e_{v_{g_{1}}, v_{g_{2}}, v_{g}}$ if and only if we have the relation $g^{-1} g_{1} g=g_{2}$ for some $g \in O$.

Conversely, to each $C$-graph $\Gamma$ one can associate a $C$-group $G_{\Gamma}=(G, Y)$ the set of $C$-generators $Y=\left\{y_{v_{i}} \mid v_{i} \in V\right\}$ of $G_{\Gamma}$ is in one to one correspondence with the set $V$ of vertices of $\Gamma$. In $G_{\Gamma}$ there is a relation $y_{v_{3}}^{-1} y_{v_{1}} y_{v_{3}}=y_{v_{2}}$ if and only if there is an edge $e_{v_{1}, v_{2}, v_{3}}$ in $\Gamma$.

Claim 4. For each $C$-graph $\Gamma$, the $C$-group $G_{\Gamma}$ is $C$-isomorphic to $G_{\Gamma_{G_{\Gamma}}}$. For each $C$-group $G$, the $C$-graphs $\Gamma_{G}$ and $\Gamma_{G_{\Gamma_{G}}}$ are isomorphic.

Proof. Obvious.

In the sequel, for a $C$-group $G_{\Gamma}$ the generators $x_{y_{v}}, v \in \Gamma$, of the semigroup $S\left(G_{\Gamma}, Y\right)$ will be denoted by $x_{v}$.

We say that a subgraph $\Gamma_{1}$ of a $C$-graph $\Gamma$ is a $C$-subgraph if $\Gamma_{1}$ is a $C$-graph.

Let $\Gamma_{1}$ be a $C$-subgraph of a $C$-graph $\Gamma$. Consider the $C$-groups $(G, Y)=G_{\Gamma}$ and $\left(G_{1}, Y_{1}\right)=G_{\Gamma_{1}}$ and their factorization semigroups $S(G, Y)$ and $S\left(G_{1}, Y_{1}\right)$. The embedding $i: \Gamma_{1} \hookrightarrow \Gamma$ defines the natural homomorphism $i_{*}: G_{\Gamma_{1}} \rightarrow G_{\Gamma}$ of $C$-groups 
and the homomorphism $i_{*}: S\left(G_{1}, Y_{1}\right) \rightarrow S(G, Y)$ of their factorization semigroups given, respectively, by $i_{*}\left(y_{v_{i}}\right)=y_{v_{i}}$ and $i_{*}\left(x_{v_{i}}\right)=x_{v_{i}}$ for $v_{i} \in \Gamma_{1} \hookrightarrow \Gamma$.

Claim 5. Let $\Gamma_{1}$ be a C-subgraph of a $C$-graph $\Gamma$. Then the homomorphism $i_{*}$ : $S\left(G_{\Gamma_{1}}, Y_{1}\right) \rightarrow S\left(G_{\Gamma}, Y\right)$ is an embedding.

Proof. Obvious.

For each $C$-graph $\Gamma$ there is a homomorphism from the $C$-group $G_{\Gamma}$ to the automorphism group of $\Gamma$. The action of $G_{\Gamma}$ is defined as follows: if $v^{\prime} \in \Gamma$ then the action of the $C$-generator $y_{v^{\prime}}$ is given by the rule: for a vertex $v$ of $\Gamma$ the image $y_{v^{\prime}}(v)$ is the head of the edge with tail $v$ and label $v^{\prime}$, for an edge $e_{v_{1}, v_{2}, v_{3}}$ the image $y_{v^{\prime}}\left(e_{v_{1}, v_{2}, v_{3}}\right)=e_{y_{v^{\prime}}\left(v_{1}\right), y_{v^{\prime}}\left(v_{2}\right), y_{v^{\prime}}\left(v_{3}\right)}$. It follows from conditions $(i)-(v)$ of the definition of $C$-graphs that this action is well defined.

In the sequel, we will consider only finitely generated $C$-groups (as groups without equipment) and $C$-graphs consisting of finitely many connected components. Denote by $m$ the number of connected components of $C$-graph $\Gamma$. Then it is easy to see that $G_{\Gamma} /\left[G_{\Gamma}, G_{\Gamma}\right] \simeq \mathbb{Z}^{m}$ and any two $C$-generators $y_{v_{1}}$ and $y_{v_{2}}$ are conjugated in the $C$ group $G_{\Gamma}$ if and only if $v_{1}$ and $v_{2}$ belong to the same connected component of $\Gamma$, that is, the set $Y$ of $C$-generators of the $C$-group $G_{\Gamma}$ is the union of $m$ conjugacy classes of $G_{\Gamma}$. Denote by ab : $G_{\Gamma} \rightarrow H_{1}\left(G_{\Gamma}, \mathbb{Z}\right)=G_{\Gamma} /\left[G_{\Gamma}, G_{\Gamma}\right]$ the natural epimorphism. In the sequel, we will assume that some numbering of the connected components of $\Gamma$ is fixed. In this case the group $H_{1}\left(G_{\Gamma}, \mathbb{Z}\right) \simeq \mathbb{Z}^{m}$ has the natural base consisting of vectors $\operatorname{ab}\left(y_{v}\right)=(0, \ldots, 0,1,0 \ldots, 0)$, where 1 stands on the $i$-th place if $v$ belongs to the $i$ th connected component of $\Gamma$. Denote the composition $a b \circ \alpha_{G_{\Gamma}}$ by $\tau$.

Let $l=l\left(v_{0} ; v_{1}, \ldots, v_{n}\right)$ be a path in a $C$-graph $\Gamma$. The number $n$ is called the length of $l$. The smallest positive integer $p_{v}$ (maybe, $n_{v}=\infty$ ), such that for any vertex $v_{i}$ of $\Gamma$ the path $l\left(v_{i} ; v, \ldots, v\right)$ of length $p_{v}$ is a loop with origin and end at $v_{i}$, is called the period of $v$. It is easy to see that $p_{v}=\min \left\{p \in \mathbb{N} \mid y_{v}^{p} \in Z\left(G_{\Gamma}\right)\right\}$, where $Z\left(G_{\Gamma}\right)$ is the center of $G_{\Gamma}$.

Claim 6. If $v_{1}$ and $v_{2}$ belong to the same connected component of a $C$-graph $\Gamma$, then $p_{v_{1}}=p_{v_{2}}$.

Proof. The elements $y_{v_{1}}$ and $y_{v_{2}}$ are conjugated in $G_{\Gamma}$. Therefore, if $y_{v_{1}}^{p_{v_{1}}} \in Z\left(G_{\Gamma}\right)$ then $y_{v_{2}}^{p_{v_{1}}}=y_{v_{1}}^{p_{v_{1}}}$ and hence $y_{v_{2}}^{p_{v_{1}}} \in Z\left(G_{\Gamma}\right)$.

Claim 7. If a vertex $v_{1}$ of a $C$-graph $\Gamma$ is such that its period $p_{v_{1}}=1$, then the $C$-group $G_{\Gamma}$ is naturally isomorphic to the direct product $G_{\Gamma_{1}} \times \mathbb{F}_{1}$, where $\mathbb{F}_{1}$ is a free group generated by $y_{v_{1}}$ and the $C$-group $G_{\Gamma_{1}}$ is generated by the all $C$-generators $y_{v}$, where $v \neq v_{1}$, and it is associated with $C$-graph $\Gamma_{1}$ obtained from $\Gamma$ deleting the vertex $v_{1}$ and the all edges labeled by $v_{1}$. 
Proof. Evident.

A $C$-group $G_{\Gamma}$ is called a finite $C$-group if the $C$-graph $\Gamma$ is a finite graph (note that a finite $C$-group is infinite in the sense of usual groups).

Let $\Gamma=\Gamma_{1} \sqcup \cdots \sqcup \Gamma_{m}$ be the decomposition into the disjoint union of the connected components of a finite $C$-graph $\Gamma$ and let $p_{i}$ be the period of the set of vertices $V_{i}=\left\{v_{i, 1}, \ldots, v_{i, n_{i}}\right\}$ of the connected component $\Gamma_{i}$. The element

$$
c=\prod_{i=1}^{m} \prod_{j=1}^{n_{i}} y_{v_{i, j}}^{p_{i}}
$$

is called the canonical element of the $C$-group $G_{\Gamma}$.

Proposition 3. Let $G_{\Gamma}$ be a finite $C$-group. Then the commutator $\left[G_{\Gamma}, G_{\Gamma}\right]$ is a finite group. Moreover, each element $g \in\left[G_{\Gamma}, G_{\Gamma}\right]$ can be written in the form

$$
g=c^{-1} \prod_{i=1}^{m} y_{i, 1}^{k_{i} p_{i}} y_{i, 1}^{a_{i, 1}} y_{i, 2}^{a_{i, 2}} \ldots y_{i, n_{i}}^{a_{i, n_{i}}},
$$

where $c$ is the canonical element of $G_{\Gamma}$ and the integers $k_{i}$ and $a_{i, j}$ satisfy the following relations and inequalities

$$
\begin{gathered}
\sum_{j=1}^{n_{i}} a_{i, j}+k_{i} p_{i}=n_{i} p_{i}, \\
0<a_{i, j} \leqslant p_{i}, \quad 0 \leqslant k_{i}<n_{i} .
\end{gathered}
$$

Proof. Applying relations (44) and since $y_{i, j}^{p_{i}}=y_{i, 1}^{p_{i}}$, each element $g \in[G, G]$ can be written in the form

$$
g=\prod_{i=1}^{m} y_{i, 1}^{k_{i} p_{i}} y_{i, 1}^{b_{i, 1}} y_{i, 2}^{b_{i, 2}} \ldots y_{i, n_{i}}^{b_{i, n_{i}}}
$$

where the integers $k_{i}$ and $b_{i, j}$ satisfy the following relations and inequalities

$$
\begin{gathered}
\sum_{j=1}^{n_{i}} b_{i, j}+k_{i} p_{i}=0, \\
\left|b_{i, j}\right| \leqslant p_{i}-1, \quad\left|k_{i}\right|<n_{i} .
\end{gathered}
$$

For fixed integers $p_{i}$ and $n_{i}$ the set of integer solutions of equations (9) under restrictions (10) is finite. Therefore $\left[G_{\Gamma}, G_{\Gamma}\right]$ is a finite group. To obtain presentation (5) from (8), it suffices to multiply presentation (8) of $g$ by $c^{-1} c$ and one more to use relations $y_{i, j}^{p_{i}}=y_{i, 1}^{p_{i}}$. 
2.2. Canonical elements of factorization semigroups. In notations used above, the element

$$
s_{\Gamma^{\prime}}=\prod_{l=1}^{k} \prod_{j=1}^{n_{i_{l}}} x_{v_{i_{l}, j}}^{p_{i_{l}}} \in S(G, Y),
$$

is well defined and it is called the canonical element associated with a subgraph $\Gamma^{\prime}=\Gamma_{i_{1}} \sqcup \cdots \sqcup \Gamma i_{k}$ of the $C$-graph $\Gamma$. If $\Gamma^{\prime}=\Gamma$, then the element $s_{\Gamma}$ is called the canonical element of the semigroup $S(G, Y)$. Obviously, $s_{\Gamma^{\prime}}$ belongs to the center of $S(G, Y)$, since each its factor $x_{v_{i_{l}, j}}^{p_{i_{l}}}$ belongs to the center of $S(G, Y)$.

An element $s_{1} \in S(G, Y)$ is said to be a divisor of an element $s \in S(G, Y)$ if there is $s_{2} \in S(G, Y)$ such that $s=s_{1} \cdot s_{2}$.

Lemma 2. An element $s \in S(G, Y)$ of length $\ln (s) \leqslant k$ is a divisor of $s_{\Gamma^{\prime}}^{k}$ if $s$ can be represented as a word in generators $x_{v_{i, j}}$, where $v_{i . j} \in \Gamma^{\prime}$.

Proof. Obvious.

Lemma 3. Let $G_{\Gamma}=(G, Y)$ be a finite $C$-group. Let the ith coordinate $\tau_{i}(s)$ of $\tau(s)$ for an element $s=s^{\prime} \cdot s^{\prime \prime} \in S(G, Y)^{G}$ is not less than $n_{i} p_{i}+1$. Assume that $\tau_{i}\left(s^{\prime \prime}\right)=0$. Then for $1 \leqslant j \leqslant n_{i}$ the element $s$ can be written in the form: $s=x_{i, j}^{p_{i}} \cdot s_{i} \cdot s^{\prime \prime}$, where $s_{i} \cdot s^{\prime \prime} \in S(G, Y)^{G}$.

Proof. Since $\tau_{i}(s) \geqslant n_{i} p_{i}+1$, there are at least $p_{i}+1$ factors $x_{v_{i, j}}$ in a factorization of $s^{\prime}, s^{\prime}=x_{v_{i_{1}, j_{1}}} \cdot \ldots \cdot x_{v_{i_{k}, j_{k}}}$ having the same $i$ and $j$. Applying relations (1), we can move them to the left and after that we obtain a new factorization $s^{\prime}=x_{v_{i, j}}^{p_{i}} \cdot\left(x_{v_{i, j}} \cdot s_{i}^{\prime}\right)$. Obviously, $s_{i} \cdot s^{\prime \prime}:=\left(x_{v_{i, j}} \cdot s_{i}^{\prime}\right) \cdot s^{\prime \prime}$ belongs to $S(G, Y)^{G}$, since $s \in S(G, Y)^{G}$. Applying Lemma 1, we complete the proof.

Corollary 1. Let $G_{\Gamma}=(G, Y)$ be a finite $C$-group. Let, for an element $s=s^{\prime} \cdot s^{\prime \prime} \in$ $S(G, Y)^{G}$, the ith coordinate $\tau_{i}(s)$ is not less than $2 n_{i} p_{i}+1$. Assume that $\tau_{i}\left(s^{\prime \prime}\right)=0$. Then the element $s$ can be written in the form: $s=s_{\Gamma_{i}} \cdot s_{i} \cdot s^{\prime \prime}$, where $s_{i} \cdot s^{\prime \prime} \in S(G, Y)^{G}$.

2.3. Ample subgraphs of $C$-graphs. Let $\Gamma^{\prime}$ be a union of some connected components of a $C$-graph $\Gamma$. We say that $\Gamma^{\prime}$ (and respectively, the union of conjugacy classes of $C$-generators of the $C$-group $G_{\Gamma}$ corresponding to $\left.\Gamma^{\prime}\right)$ is ample if any two vertices of $\Gamma$ can be connected by a path along edges of $\Gamma$ labeled by vertices belonging to $\Gamma^{\prime}$. In language of $C$-groups, it means that any two conjugated $C$-generators of $G_{\Gamma}$ are conjugated by some element of the subgroup $G_{\Gamma^{\prime}}$ of $G_{\Gamma}$ generated by the $C$-generators $y_{v}, v \in \Gamma^{\prime}$. Note that $G_{\Gamma^{\prime}}$ is the image of the $C$-group $G_{\widetilde{\Gamma}^{\prime}}$ under the $C$-homomorphism $i_{*}: G_{\widetilde{\Gamma}^{\prime}} \rightarrow G_{\Gamma}$ given by embedding $i: \widetilde{\Gamma}^{\prime} \hookrightarrow \Gamma$, where $\widetilde{\Gamma}^{\prime}$ is the $C$-subgraph of $\Gamma$ obtained from $\Gamma^{\prime}$ after deleting all vertices $v \notin \Gamma^{\prime}$ and all edges labeled by the vertices $v \notin \Gamma^{\prime}$.

The $C$-group $G_{\Gamma}$ acts on $\Gamma$. Therefore the homomorphism $i_{*}$ defines an action of $G_{\widetilde{\Gamma}^{\prime}}$ on $\Gamma$ leaving fixed each connected component of $\Gamma$. It is easy to see that if $\Gamma^{\prime}$ is ample then $G_{\widetilde{\Gamma}^{\prime}}$ acts transitively on the set of vertices of each connected component of $\Gamma$. 
Lemma 4. Let a union $\Gamma^{\prime}$ of some connected components of a finite $C$-graph $\Gamma$ is ample and let $v_{o}, v_{e}$ be two vertices belonging to a connected component, say $\Gamma_{1}$, of $\Gamma$. Then there is a path $l\left(v_{o} ; v_{1}, \ldots v_{n}\right)$ connecting $v_{o}$ and $v_{e}$ such that $v_{1}, \ldots, v_{n}$ are vertices of $\Gamma^{\prime}$.

Proof. Let $V_{1}$ be the set of vertices of $\Gamma_{1}$. For $v \in V_{1}$ denote by $V_{1}(v)$ the set of all vertices $v^{\prime} \in V_{1}$ such that for $v^{\prime}$ there is a path $l\left(v ; v_{1}, \ldots v_{n}\right)$ connecting $v$ and $v^{\prime}$ such that $v_{1}, \ldots, v_{n}$ are vertices of $\Gamma^{\prime}$. We say that $v \leqslant v^{\prime}$ if $v^{\prime} \in V_{1}(v)$. It is easy to see that $V_{1}(v) \subset V_{1}\left(v^{\prime}\right)$ if $v^{\prime} \leqslant v$. Therefore the set of subsets $V_{1}(v) \subset V_{1}, v \in V_{1}$, is partially ordered under inclusions and hence there is a maximal one, say $V_{1}(\tilde{v})$, since $\Gamma$ is a finite graph.

Next, it is easy to see that the $C$-group $G_{\widetilde{\Gamma}^{\prime}}$ acts on the set of subsets $V_{1}(v) \subset V_{1}$, $v \in V_{1}$, by the rule: $y_{v_{1}}\left(V_{1}(v)\right)=V_{1}\left(y_{v_{1}}(v)\right)$ for a $C$-generator $y_{v_{1}} \in G_{\widetilde{\Gamma}^{\prime}}$. Therefore for a maximal subset $V_{1}(\tilde{v})$ we have $V_{1}(\tilde{v})=V_{1}(v)$ for all $v \in V_{1}(\tilde{v})$, since $G_{\widetilde{\Gamma}^{\prime}}$ acts transitively on $V_{1}$. Hence, $V_{1}$ can be represented as the disjoint union $V_{1}\left(\tilde{v}_{1}\right) \sqcup \cdots \sqcup V_{1}\left(\tilde{v}_{m}\right)$ of maximal subsets $V_{1}\left(\tilde{v}_{i}\right)$. Finally, since $\Gamma^{\prime}$ is ample, we obtain that $m=1$.

Let a union $\Gamma^{\prime}$ of some connected components of a finite $C$-graph $\Gamma$ be ample and let $\Gamma_{1}$ be a connected component of $\Gamma$. By definition, the distance $d_{\Gamma^{\prime}}\left(v_{o}, v_{e}\right)$ between two vertices $v_{o}, v_{e}$ of $\Gamma_{1}$ with respect to $\Gamma^{\prime}$ is the smallest $n$ such that there is a path $l\left(v_{o} ; v_{1}, \ldots v_{n}\right)$ connecting $v_{o}$ and $v_{e}$ such that $v_{1}, \ldots, v_{n}$ are vertices of $\Gamma^{\prime}$. The number $d_{\Gamma^{\prime}}\left(\Gamma_{1}\right)=\max _{v_{o}, v_{e} \in V_{1}} d_{\Gamma^{\prime}}\left(v_{o}, v_{e}\right)$ is called the diameter of $\Gamma_{1}$ with respect to $\Gamma^{\prime}$.

Proposition 4. Let $G_{\Gamma}=(G, Y)$ be a finite C-group, $\Gamma^{\prime}=\Gamma_{1} \sqcup \cdots \sqcup \Gamma_{k}$ a ample subgraph of $\Gamma$, and $\Gamma \backslash \Gamma^{\prime}=\Gamma_{k+1} \sqcup \cdots \sqcup \Gamma_{m}$, where $\Gamma_{i}, i=1, \ldots, m$, are the connected components of $\Gamma$. Denote by $d=d_{\Gamma^{\prime}}=\max \left(d_{\Gamma^{\prime}}\left(\Gamma_{1}\right), \ldots, d_{\Gamma^{\prime}}\left(\Gamma_{m}\right)\right)$. Let an element $s \in$ $S(G, Y)^{G}$ is such that $\tau_{i}(s) \geqslant 2 n_{i} p_{i} d+1$ for all $i \leqslant k$, where $\tau_{i}(s)$ is the ith coordinate of $\tau(s)$. Then the element $s$ can be written in the form: $s=\left(x_{v_{k+1,1}}^{a_{k+1}} \cdot \ldots \cdot x_{v_{m, 1}}^{a_{m}}\right) \cdot s_{\Gamma^{\prime}}^{d} \cdot s_{1}$, where $a_{i}=\tau_{i}(s)$ for $i=k+1, \ldots, m$ and $s_{1} \in S(G, Y)$ is such that $\tau_{i}\left(s_{1}\right)=0$ for $i=k+1, \ldots, m$.

Proof. Let us write the element $s$ in the form: $s=s^{\prime} \cdot s^{\prime \prime}$ where $s^{\prime}$ and $s^{\prime \prime}$ are such that $\tau_{i}\left(s^{\prime}\right)=0$ for $i \geqslant k+1$ and $\tau_{i}\left(s^{\prime \prime}\right)=0$ for $i \leqslant k$. Then, by Corollary 1, the element $s$ can be written in the form: $s=\widetilde{s}^{\prime} \cdot s_{\Gamma^{\prime}}^{d} \cdot s^{\prime \prime}$.

Let for some $j$ a letter $x_{v_{m, j}}$ enter in $s^{\prime \prime}$. Connect the vertex $v_{m, 1}$ with $v_{m, j}$ by a path $l\left(v_{m, 1} ; v_{1}, \ldots, v_{r}\right)$ of length $r \leqslant d$ (remind that $\Gamma^{\prime}$ is ample, therefore by Lemma 4. $v_{m, 1}$ and $v_{m, j}$ can be connected by such a path), where $v_{1}, \ldots, v_{r}$ are some vertices of $\Gamma^{\prime}$, and write $s^{\prime \prime}$ in the form $s^{\prime \prime}=x_{v_{m, j}} \cdot \widetilde{s}^{\prime \prime}$. By Lemma 2, $s_{\Gamma^{\prime}}^{d}=\widetilde{s} \cdot\left(x_{v_{1}} \cdot \ldots \cdot x_{v_{r}}\right)$ for some $\widetilde{s}$. We have $\alpha\left(x_{v_{1}} \cdot \ldots \cdot x_{v_{r}}\right)=y_{v_{1}} \ldots y_{v_{r}}$ and by definition of $C$-graphs of $C$-groups, we have $\left(y_{v_{1}} \ldots y_{v_{r}}\right) y_{v_{m, j}}\left(y_{v_{1}} \ldots y_{v_{r}}\right)^{-1}=y_{v_{m, 1}}$. Therefore,

$$
s_{\Gamma^{\prime}}^{d} \cdot x_{v_{m, j}}=\widetilde{s} \cdot\left(x_{v_{1}} \cdot \ldots \cdot x_{v_{r}}\right) \cdot x_{v_{m, j}}=\widetilde{s} \cdot x_{v_{m, 1}} \cdot\left(x_{v_{1}} \cdot \ldots \cdot x_{v_{r}}\right)
$$

and hence (after moving $x_{v_{m, 1}}$ to the right)

$$
s=s_{1}^{\prime} \cdot s_{\Gamma^{\prime}}^{d} \cdot s^{\prime \prime}=\widetilde{s} \cdot \widetilde{s} \cdot x_{v_{m, 1}} \cdot\left(x_{v_{1}} \cdot \ldots \cdot x_{v_{r}}\right) \cdot \widetilde{s}^{\prime \prime}=s_{1}^{\prime} \cdot s_{1}^{\prime \prime} \cdot x_{v_{m, 1}},
$$


where $s_{1}^{\prime}$ is such that $\tau_{i}\left(s_{1}^{\prime}\right)=0$ for $i \geqslant k+1$ and $\tau_{i}\left(s_{1}^{\prime}\right) \geqslant 2 n_{i} p_{i} d+1$ for all $i \leqslant k$, and $s_{1}^{\prime \prime}$ is such that $\tau_{i}\left(s_{1}^{\prime \prime}\right)=0$ for $i \leqslant k$.

If again for some $j$ a letter $x_{v_{m, j}}$ enters in $s_{1}^{\prime \prime}$, applying Corollary 1, Claim 4, and Lemma 2, we can repeat the transformation described above, and so on, and we obtain a factorization $s=s_{a_{m}}^{\prime} \cdot s_{a_{m}}^{\prime \prime} \cdot x_{v_{m, 1}}^{a_{m}}$. After that we can repeat the transformation described above and we obtain a factorization $s=s_{a_{m}+a_{m-1}}^{\prime} \cdot s_{a_{m}+a_{m-1}}^{\prime \prime} \cdot x_{v_{m-1,1}}^{a_{m-1}} \cdot x_{v_{m, 1}}^{a_{m}}$, and so on. After the last step of these transformations, we move the obtained product $\left(x_{v_{k+1,1}}^{a_{k+1}} \cdot \ldots \cdot x_{v_{m, 1}}^{a_{m}}\right)$ to the left and apply Corollary 1 to complete the proof.

2.4. $C$-graphs of equipped groups. To each equipped group $(G, O)$, one can associate a $C$-graph. By definition, the $C$-graph $\Gamma=\Gamma_{(G, O)}$ of an equipped group $(G, O)$ is the $C$-graph whose set of vertices is in one to one correspondence with the set $O$. Two vertices $v_{g_{1}}$ and $v_{g_{2}}, g_{1}, g_{2} \in O$ are connected by the labeled edge $e_{v_{g_{1}}, v_{g_{2}}, v_{g}}$ if and only if $g^{-1} g_{1} g=g_{2}$ for some $g \in O$.

Example 1. To describe the $C$-graph $\Gamma_{\left(\Sigma_{n}, T_{n}\right)}$ of the equipped symmetric group $\left(\Sigma_{n}, T_{n}\right)$, where $T_{n}$ is the set of transpositions, consider a $(n-1)$-simplex $\Delta_{n-1}$. Let $V_{1}, \ldots V_{n}$ be the vertices of $\Delta_{n-1}$ and $E_{i, j}$ its edges connecting the vertices $V_{i}$ and $V_{j}$. The vertices $v_{i, j}$ of $\Gamma_{\left(\Sigma_{n}, T_{n}\right)}$ are the middles of the edges $E_{i, j}$. If $E_{i, j}$ and $E_{k, l}$ are skewed edges, then the edge of $\Gamma_{\left(\Sigma_{n}, T_{n}\right)}$ with tail $v_{i, j}$ and label $v_{k, l}$ is the loop. The head of the edge with tail $v_{i, j}$ and label $v_{j, k}$ is $v_{i, k}$.

To each equipped group $(G, O)$, we can associate a $C$-group $G_{\Gamma_{(G, O)}}$. Denote the $C$-generators $y_{v_{g_{i}}}, g_{i} \in O$, of the $C$-group $G_{\Gamma_{(G, O)}}$ by $y_{g_{i}}$. We have the natural homomorphism of equipped groups $\beta=\beta_{(G, O)}: G_{\Gamma_{(G, O)}} \rightarrow(G, O)$ given by $\beta\left(y_{g_{i}}\right)=g_{i}$ for all $g_{i} \in O$. Obviously, $\beta_{\mid Y}: Y \rightarrow O$ is one to one correspondence.

Claim 8. (i) $\operatorname{ker} \beta$ is a subgroup of the center of $G_{\Gamma_{(G, O)}}$.

(ii) If $(G, O)$ is an equipped group such that $G$ is generated by the elements of $O$, then $\beta$ and $\beta_{\mid\left[G_{\Gamma(G, O)}, G_{\Gamma_{(G, O)}}\right]}:\left[G_{\Gamma_{(G, O)}}, G_{\Gamma_{(G, O)}}\right] \rightarrow[G, G]$ are epimorphisms.

Proof. Obvious.

2.5. Equivalence of equipped groups. Let $\left(G_{1}, O_{1}\right)$ and $\left(G_{2}, O_{2}\right)$ be two equipped groups such that $G_{1}$ and $G_{2}$ are generated, resp., by the elements of $O_{1}$ and $O_{2}$. We say that $\left(G_{1}, O_{1}\right)$ and $\left(G_{2}, O_{2}\right)$ are equivalent if the $C$-graphs $\Gamma_{\left(G_{1}, O_{1}\right)}$ and $\Gamma_{\left(G_{2}, O_{2}\right)}$ are isomorphic as $C$-graphs.

Claim 9. Let $\left(G_{1}, O_{1}\right)$ and $\left(G_{2}, O_{2}\right)$ be two equivalent equipped groups. Then the C-groups $G_{\Gamma_{\left(G_{1}, O_{1}\right)}}$ and $G_{\Gamma_{\left(G_{2}, O_{2}\right)}}$ are $C$-isomorphic.

Proof. It follows from Claim 4 .

It follows from Claims 8 and 4 that for each class of equivalent equipped groups corresponding to a $C$-graph $\Gamma$, there is a maximal one, namely, the $C$-group $G_{\Gamma}$, such that for any equipped group $(G, O)$ belonging to this class there is an epimorphism of 
equipped groups, namely $\beta_{(G, O)}: G_{\Gamma_{(G, O)}} \rightarrow(G, O)$, which is defined uniquely by an isomorphism $\Gamma \simeq \Gamma_{(G, O)}$ and by the following condition: for each $C$-generator $y$ of $G_{\Gamma}$ the image $\beta_{(G, O)}(y)=g \in O$ if $y$ and $g$ correspond to the same vertex $v$ of $\Gamma \simeq \Gamma_{(G, O)}$. By Claim 8 , ker $\beta_{(G, O)}$ is a subgroup of the center $Z\left(G_{\Gamma}\right)$ of $G_{\Gamma}$. The inverse statement is also true, namely, an equipped group $(G, O)$, obtained as the quotient group $G_{\Gamma} / H$ of a $C$-group $G_{\Gamma}$, is equivalent to $G_{\Gamma}$ if $H$ is a subgroup of $Z\left(G_{\Gamma}\right)$ and it contains neither $C$-generators of $G_{\Gamma}$ nor quotients $y_{i} y_{j}^{-1}$ of $C$-generators $y_{i}$ and $y_{j}, y_{i} \neq y_{j}$.

The order $a_{(G, O)}=\left|H \cap\left[G_{\Gamma}, G_{\Gamma}\right]\right|$ of the group $H \cap\left[G_{\Gamma}, G_{\Gamma}\right]$ is called the ambiguity index of the equipped group $\left(G=G_{\Gamma} / H, O\right)$ equivalent to $G_{\Gamma}$.

Proposition 5. Let an equipped group $(G, O)$ is equivalent to a $C$-group $G_{\Gamma}$. If $G$ is a perfect group, then $G_{\Gamma}$ is isomorphic to the direct product $\left[G_{\Gamma}, G_{\Gamma}\right] \times\left(G_{\Gamma} /\left[G_{\Gamma}, G_{\Gamma}\right]\right)$.

Proof. For each connected component $\Gamma_{i}$ of $\Gamma$, let us choose a vertex $v_{i} \in \Gamma_{i}$.

The restriction of $\beta_{(G, O)}$ to $\left[G_{\Gamma}, G_{\Gamma}\right]$ is an epimorphism onto $G$, since $\beta_{(G, O)}$ is epimorphism and $G$ is a perfect group. Therefore for each $C$-generator $y_{v_{i}}$ of $G_{\Gamma}$, there is an element $g_{i} \in\left[G_{\Gamma}, G_{\Gamma}\right]$ such that $\beta_{(G, O)}\left(g_{i}\right)=\beta_{(G, O)}\left(y_{v_{i}}\right)$. We have $y_{v_{i}} g_{i}^{-1} \in$ $\operatorname{ker} \beta_{(G, O)} \subset Z\left(G_{\Gamma}\right)$ and $a b\left(y_{v_{i}} g_{i}^{-1}\right)=(0, \ldots, 0,1,0, \ldots, 0)$, where 1 stands on the $i$ th place. Therefore the elements $y_{v_{i}} g_{i}^{-1}$ generate in $Z\left(G_{\Gamma}\right)$ a free abelian group $H$ such that $a b_{\mid H}: H \rightarrow G_{\Gamma} /\left[G_{\Gamma}, G_{\Gamma}\right]$ is an isomorphism and Proposition follows from the short exact sequence

$$
1 \rightarrow\left[G_{\Gamma}, G_{\Gamma}\right] \rightarrow G_{\Gamma} \rightarrow G_{\Gamma} /\left[G_{\Gamma}, G_{\Gamma}\right] \rightarrow 1 .
$$

Proposition 6. Let an equipped group $(G, O)$ is equivalent to a $C$-group $G_{\Gamma}$. If $O$ consists of a single conjugasy class, then the group $G_{\Gamma}$ is isomorphic to the semidirect product $\left[G_{\Gamma}, G_{\Gamma}\right] \rtimes \mathbb{Z}$.

Proof. It follows from exact sequence (11), since $G_{\Gamma} /\left[G_{\Gamma}, G_{\Gamma}\right] \simeq \mathbb{Z}$ if $O$ consists of a single conjugacy class.

Note that Proposition 6 is not true if $O$ consists of more than one conjugacy class. For example, Proposition [6] is not true if $G_{\Gamma}$ is a free group $\mathbb{F}^{n}, n>1$, whose set of $C$-generators is the union of conjugacy classes of a set of free generators of $\mathbb{F}^{n}$.

Lemma 5. Let a subgroup $H$ of the center $Z\left(G_{\Gamma}\right)$ of a finite $C$-group $G_{\Gamma}$ be generated by the elements $y_{v_{i}}^{k_{i} p_{i}} \in Z\left(G_{\Gamma}\right), i=1, \ldots, m$, where $k_{i} p_{i} \geqslant 2$, $v_{i}$ is a vertex of the $i$ th connected component of the $C$-graph $\Gamma$ and $p_{i}$ is its period. Then $(G, O)=G_{\Gamma} / H$ is an equipped group equivalent to $G_{\Gamma}$ and $\beta_{(G, O)}:\left[G_{\Gamma_{(G, O)}}, G_{\Gamma_{(G, O)}}\right] \rightarrow[G, G]$ is an isomorphism. In particular, $a_{(G, O)}=1$.

Proof. Obvious. 
2.6. The type homomorphism. Let $(G, O)$ be an equipped group, $\Gamma=\Gamma_{(G, O)}$ its $C$-graph, and $G_{\Gamma}$ the $C$-group equivalent to $(G, O)$. The homomorphism $\beta=\beta_{(G, O)}$ : $G_{\Gamma} \rightarrow(G, O)$ defines a homomorphism of semigroups $\beta_{*}: S\left(G_{\Gamma}, Y\right) \rightarrow S(G, O)$ given by $\beta_{*}\left(x_{y_{g}}\right)=x_{g}$ for $g \in O$.

Claim 10. The homomorphism $\beta_{*}$ is an isomorphism.

Proof. Obvious.

In the sequel, according to Claim 10, we will identify the semigroups $S(G, O)$ and $S\left(G_{\Gamma_{(G, O)}}, Y\right)$.

The homomorphism of semigroups $\tau: S(G, O)=S\left(G_{\Gamma_{(G, O)}}, Y\right) \rightarrow \mathbb{Z}_{\geqslant 0}^{m} \subset \mathbb{Z}^{m}=$ $G_{\Gamma_{(G, O)}} /\left[G_{\Gamma_{(G, O)}}, G_{\Gamma_{(G, O)}}\right]$ is called the type homomorphism and the image $\tau(s)$ of $s \in$ $S(G, O)$ is called the type of $s$. If $O$ consists of a single conjugacy class, then the homomorphism $\tau$ can (and will) be identified with the homomorphism $\ln : S(G, O) \rightarrow$ $\mathbb{Z}_{\geqslant 0}$. In general case, if $\tau(s)=\left(\tau_{1}(s), \ldots, \tau_{m}(s)\right)$, then $\ln (s)=\sum_{i=1}^{m} \tau_{i}(s)$.

An element $g \in G_{\Gamma}$ is called positive if there is $s \in S\left(G_{\Gamma}, Y\right)$ such that $\alpha_{G_{\Gamma}}(s)=g$.

Lemma 6. Any element $g$ of the $C$-group $G_{\Gamma}$ can be represented in the form:

$$
g=g_{1} g_{2}^{-1}
$$

where $g_{1}$ are $g_{2}$ are positive elements. In particular, $g \in\left[G_{\Gamma}, G_{\Gamma}\right]$ if and only if $a b\left(g_{1}\right)=a b\left(g_{2}\right)$ in representation (12) of $g$ as a quotient of two positive elements $g_{1}$ and $g_{2}$.

If $G_{\Gamma}$ is a finite $C$-group, then for each $g \in G_{\Gamma}$ there is a presentation (12) of $g$ as a quotient of two positive elements $g_{1}$ and $g_{2}$ such that $g_{2}=\alpha_{G_{\Gamma}}\left(s_{\Gamma}^{n}\right)$ for some $n$ and $g_{1}=\alpha_{G_{\Gamma}}\left(s_{1}\right)$ for some $s_{1} \in S\left(G_{\Gamma}, Y\right)^{G_{\Gamma}}$, where $s_{\Gamma}$ is the canonical element of $S\left(G_{\Gamma}, Y\right)$.

Proof. The first part of Lemma 6 is obvious. The second part follows from Lemma 2.

Let $H$ be a subgroup of the center of a finite $C$-group $G_{\Gamma}$. Denote by $z_{H}$ the minimal exponent $n$ such that each element $g \in H \cap\left[G_{\Gamma}, G_{\Gamma}\right]$ has presentation (12) in which $g_{2}=\alpha_{G_{\Gamma}}\left(s_{\Gamma}^{n}\right)$.

Proposition 7. Let $G_{\Gamma}=(G, Y)$ be a finite C-group, $\Gamma^{\prime}=\Gamma_{1} \sqcup \cdots \sqcup \Gamma_{k} \subset \Gamma$ be an ample subgraph of $\Gamma$, and $\widetilde{\Gamma^{\prime}}$ the $C$-graph defined by $\Gamma^{\prime}$. Then $i_{* \mid\left[G_{\widetilde{\Gamma^{\prime}}}, G_{\widetilde{\Gamma^{\prime}}}\right]}:\left[G_{\widetilde{\Gamma^{\prime}}}, G_{\widetilde{\Gamma^{\prime}}}\right] \rightarrow$ $\left[G_{\Gamma}, G_{\Gamma}\right]$ is an epimorphism.

Proof. It follows from Proposition 4 and Lemma 6.

Corollary 2. Let $G_{\Gamma}$ and $G_{\widetilde{\Gamma}^{\prime}}$ be finite $C$-groups equivalent respectively to equipped groups $(G, O)$ and $\left(G, O^{\prime}\right)$, where $O^{\prime} \subset O$ are equipments of $G$ such that the elements of $O^{\prime}$ generate the group $G$. Then $a_{(G, O)} \leqslant a_{\left(G, O^{\prime}\right)}$, in particular, if $a_{\left(G, O^{\prime}\right)}=1$, then $a_{(G, O)}=1$. 


\section{Stability of FACTORIZATion SEMigroups}

3.1. Equivalence of elements. Elements $s_{1}$ and $s_{2}$ of a semigroup $S$ are said to be $r$-equivalent (resp., l-equivalent) if there is an element $s_{3} \in S$ such that $s_{1} \cdot s_{3}=s_{2} \cdot s_{3}$ (resp., $s_{3} \cdot s_{1}=s_{3} \cdot s_{2}$ ), and they are equivalent if there are two elements $s_{3}, s_{4} \in S$ such that $s_{3} \cdot s_{1} \cdot s_{4}=s_{3} \cdot s_{2} \cdot s_{4}$. Notation $s_{1} \sim s_{2}$ (resp., $s_{1} \sim_{r} s_{2}$ and $s_{1} \sim_{l} s_{2}$ ) means that elements $s_{1}$ and $s_{2}$ are equivalent (resp., $r$-equivalent and $l$-equivalent). It is easy to see that if $s_{1} \sim s_{2}$, where $s_{1}, s_{2} \in S(G, O)$, then $\tau\left(s_{1}\right)=\tau\left(s_{2}\right)$.

Lemma 7. Let $s_{1}, s_{2} \in S(G, O)$ be two elements of a factorization semigroup over a group $G$. Then the following statements are equivalent:

(i) $s_{1} \sim_{r} s_{2}$;

(ii) $s_{1} \sim_{l} s_{2}$

(iii) $s_{1} \sim s_{2}$.

Proof. We prove only the implication $(i) \Rightarrow(i i)$, since the proof of all other implications are similar.

We have $s_{1} \cdot s_{3}=\rho\left(\alpha_{G}\left(s_{1}\right)\right)\left(s_{3}\right) \cdot s_{1}$ and $s_{2} \cdot s_{3}=\rho\left(\alpha_{G}\left(s_{2}\right)\right)\left(s_{3}\right) \cdot s_{2}$. Since $\alpha_{G}$ is a homomorphism, it is easy to see that $\alpha_{G}\left(s_{1}\right)=\alpha_{G}\left(s_{2}\right)$ if $s_{1}$ and $s_{2}$ are $r$-equivalent (resp., l-equivalent or equivalent). Therefore, if $s_{1} \sim_{r} s_{2}$, that is, $s_{1} \cdot s_{3}=s_{2} \cdot s_{3}$ for some $s_{3} \in S(G, O)$, then $\rho\left(\alpha_{G}\left(s_{1}\right)\right)\left(s_{3}\right) \cdot s_{1}=\rho\left(\alpha_{G}\left(s_{2}\right)\right)\left(s_{3}\right) \cdot s_{2}$, that is, $s_{1} \sim_{l} s_{2}$.

Lemma 8. Let $S(G, O)$ be a factorization semigroup over a group $G$. Then the relation $s_{1} \sim s_{2}$ is an equivalence relation.

Proof. Let we have $s_{1} \sim s_{2}$ and $s_{2} \sim s_{3}$. Then, by Lemma 7, there are elements $s_{4}$ and $s_{5}$ such that $s_{4} \cdot s_{1}=s_{4} \cdot s_{2}$ and $s_{2} \cdot s_{5}=s_{3} \cdot s_{5}$. Therefore $s_{4} \cdot s_{1} \cdot s_{5}=s_{4} \cdot s_{2} \cdot s_{5}=s_{4} \cdot s_{3} \cdot s_{5}$, that is, $s_{1} \sim s_{3}$.

Lemma 9. Let $s_{1}, s_{2}, s_{3}, s_{4} \in S(G, O)$ be four elements of a factorization semigroup over a group $G$. Then $s_{1} \cdot s_{2} \sim s_{3} \cdot s_{4}$ if $s_{1} \sim s_{3}$ and $s_{2} \sim s_{4}$. If $s_{1} \cdot s_{3} \sim s_{2} \cdot s_{4}$ and $s_{1} \sim s_{2}$, then $s_{3} \sim s_{4}$.

Proof. Similar to the proof of Lemma 8.

Theorem 2. Let $G$ be a $C$-group and $Y$ the set of its $C$-generators. Two elements $s_{1}$ and $s_{2} \in S(G, Y)$ are equivalent if and only if $\alpha_{G}\left(s_{1}\right)=\alpha_{G}\left(s_{2}\right)$, where $\alpha_{G}$ is the product homomorphism.

Proof. It is obvious that if $s_{1} \sim s_{2}$, then $\alpha_{G}\left(s_{1}\right)=\alpha_{G}\left(s_{2}\right)$.

Denote by $x_{i}=x_{y_{i}}$ the generator of $S(G, Y)$ corresponding to the $C$-generator $y_{i} \in Y$. Let $s_{1}=x_{i_{1}} \cdot \ldots \cdot x_{i_{n}}$ and $s_{2}=x_{j_{1}} \cdot \ldots \cdot x_{j_{k}}$ be such that $\alpha_{G}\left(s_{1}\right)=$ $y_{i_{1}} \ldots y_{i_{n}}=y_{j_{1}} \ldots y_{j_{m}}=\alpha_{G}\left(s_{2}\right)$. Then it is easy to see that $\tau\left(s_{1}\right)=\tau\left(s_{2}\right)$ and, in 
particular, $n=k$. In addition, the word $w=y_{i_{1}} \ldots y_{i_{n}} y_{j_{n}}^{-1} \ldots y_{j_{1}}^{-1}$ in letters of $Y$ represents the unity of $G$.

To prove $s_{1} \sim s_{2}$ we will use some admissible transformations of van Kampen diagrams defined over the $C$-presentation of $G$. To define them, recall that by van Kampen Lemma (see, for example, [8] or [9]), for the word $w$ there is a van Kampen diagram, that is, a planar finite cell complex $D \subset \mathbb{R}^{2}$ with the following additional data and satisfying the following additional properties:

1. The complex $D$ is connected and simply connected.

2. Each edge (one-cell) of $D$ is directed and labeled by a letter $y \in Y$.

3. Some vertex (zero-cell) which belongs to the topological boundary $\partial D$ of $D$ is specified as a base-vertex $A$ called the origin of the diagram.

4. Each region (two-cell) of $D$ is a quadrangle corresponding to a $C$-relation $y_{i} y_{j}=$ $y_{k} y_{i}$ of the $C$-presentation of $G$ (see Fig. 1 ; the vertex $v_{1}$ will be called the bottom of $Q$ and the vertex $v_{2}$ will be called the top of $Q$ ).

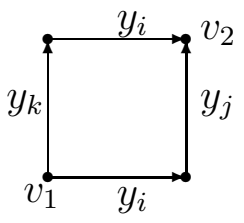

Fig.1

5. The boundary cycle $\partial D$, that is, an edge-path corresponding to going around once in the clockwise direction along the boundary of the unbounded complementary region of $D$, starting and ending at the origin $A$, has the label $w=y_{i_{1}} \ldots y_{i_{n}} y_{j_{n}}^{-1} \ldots y_{j_{1}}^{-1}$.

Since in our case the word $w$ splits into two subwords: the first one consists of letters with positive exponents and the other one consists of letters with negative exponents, the van Kampen diagram $D$ is of the following form: it is a chain of discs connected by simple directed paths (see Fig. 2).

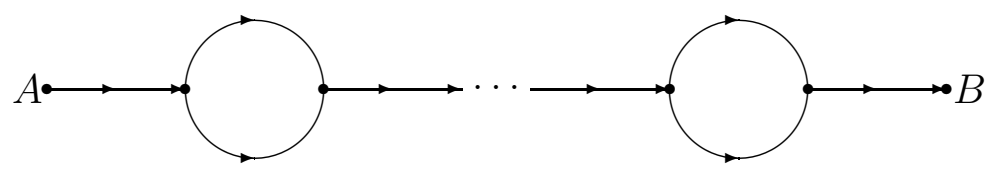

Fig.2

Denote the end vertex of this chain by $B$ and call it the end of $D$. Diagrams satisfying conditions $1-5$ will be called admissible.

It follows from Lemmas 8 and 9 that it suffices to consider only the case when $D$ consists of a single disc (see Fig. 3). 


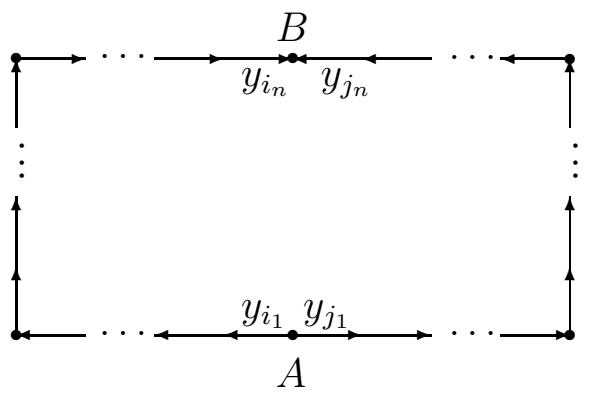

Fig.3

A vertex $v$ in a van Kampen diagram $D$ is called locally maximal (resp., minimal), if there is not an edge of $D$ for which $v$ is the tail (resp., head). A path $l$ along edges $e_{1}, \ldots, e_{k}$ of $D$ is called increasing if the tail of each edge $e_{i+1}$ is the head of the edge $e_{i}$ for $i=1, \ldots, k$. The label of increasing path $l$ is a positive word $y_{l_{1}} \ldots y_{l_{k}}$.

The origin $A$ and the end $B$ divides the boundary $\partial D$ into two parts. The increasing path $\partial_{l} D$ (resp., $\partial_{r} D$ ) along the boundary $\partial D$ connecting $A$ and $B$ and having the label $y_{i_{1}} \ldots y_{i_{n}}$ (resp., $y_{j_{1}} \ldots y_{j_{n}}$ ) will be called the left (resp., right) side of $\partial D$.

If the origin $A$ is not a locally minimal vertex of $D$, then there are a locally minimal vertex $C$ and a simple increasing path $l$ connecting the vertices $C$ and $A$. (Note that the vertex $C$ can not be a vertex belonging to the boundary $\partial D$, since a positive word in $C$-generators can not represent the unity of a $C$-group.) Then we can cut the disc $D$ along the path $l$ and, as a result, we obtain a new disc diagram $D^{\prime}$ (see Fig. 4) in which $C$ is the origin and the label of $\partial D^{\prime}$ is

$$
y_{l_{1}} \ldots y_{l_{k}} y_{i_{1}} \ldots y_{i_{n}} y_{j_{n}}^{-1} \ldots y_{j_{1}}^{-1} y_{l_{k}}^{-1} \ldots y_{l_{1}}^{-1}
$$

where $y_{l_{1}} \ldots y_{l_{k}}$ is the label of $l$.
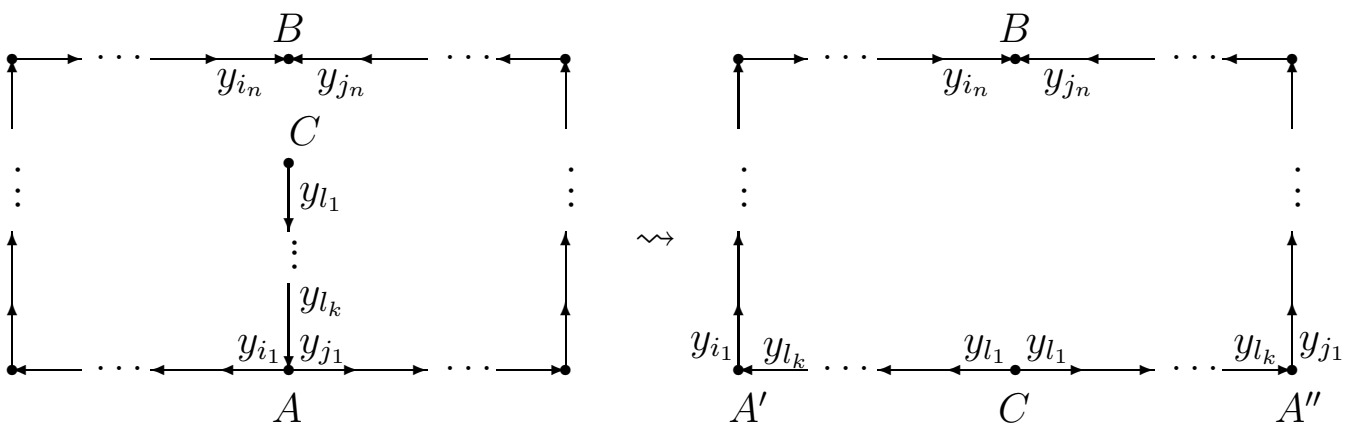

Fig.4

We call the transformation $D \rightsquigarrow D^{\prime}$ described above an admissible transformation $I$. Note that after transformation $I$ the diagram $D^{\prime}$ is admissible and the origin $C$ of $D^{\prime}$ is a locally minimal vertex. 
Similarly, if the end $B$ is not a locally maximal vertex of $D$, then there are a locally maximal vertex $C$ and a simple increasing path $l$ connecting $B$ and $C$. Then we can cut the disc $D$ along the path $l$ and, as a result, we obtain a new admissible disc diagram $D^{\prime}$ in which $C$ is the end and the label of $\partial D^{\prime}$ is

$$
y_{i_{1}} \ldots y_{i_{n}} y_{l_{1}} \ldots y_{l_{k}} y_{l_{k}}^{-1} \ldots y_{l_{1}}^{-1} y_{j_{n}}^{-1} \ldots y_{j_{1}}^{-1}
$$

where $y_{l_{1}} \ldots y_{l_{k}}$ is the label of $l$. We call this transformation $D \rightsquigarrow D^{\prime}$ an admissible transformation $I I$. Note that after transformation $I I$ the end $C$ of $D^{\prime}$ is a locally maximal vertex.

Let $v \in \partial_{l} D$ be a vertex of an admissible disc diagram $D$ such that $v$ is neither the origin $A$ nor the end $B$ of $D$ and there is an edge $e$ of $D, e \not \subset \partial D$, for which $v$ is the tail. Denote by $y_{0}$ the label of this edge and let $C$ be its head, $C \notin \partial D$. Assume for definiteness that $v \in \partial_{l} D$ (the case when $v \in \partial_{r} D$ is similar). Let $y_{i_{k}}$ (resp., $y_{i_{k+1}}$ ) be the label of the edge belonging to $\partial D$ for which $v$ is the head (resp., the tail). Let us cut the diagram $D$ along $e$ and after that paste sequentially $n-k$ additional quadrangles as it is depicted in Fig. 5, where the quadrangle, glued at the $l$ th step to the cut disc diagram along the edges labeled by $y_{0}$ and $y_{i_{k+l}}$, corresponds to the relation $y_{i_{k+l}} y_{0}=y_{0} y_{i_{k+l}}^{\prime}, l=k+1, \ldots, n-k$, in the $C$-group $G$.
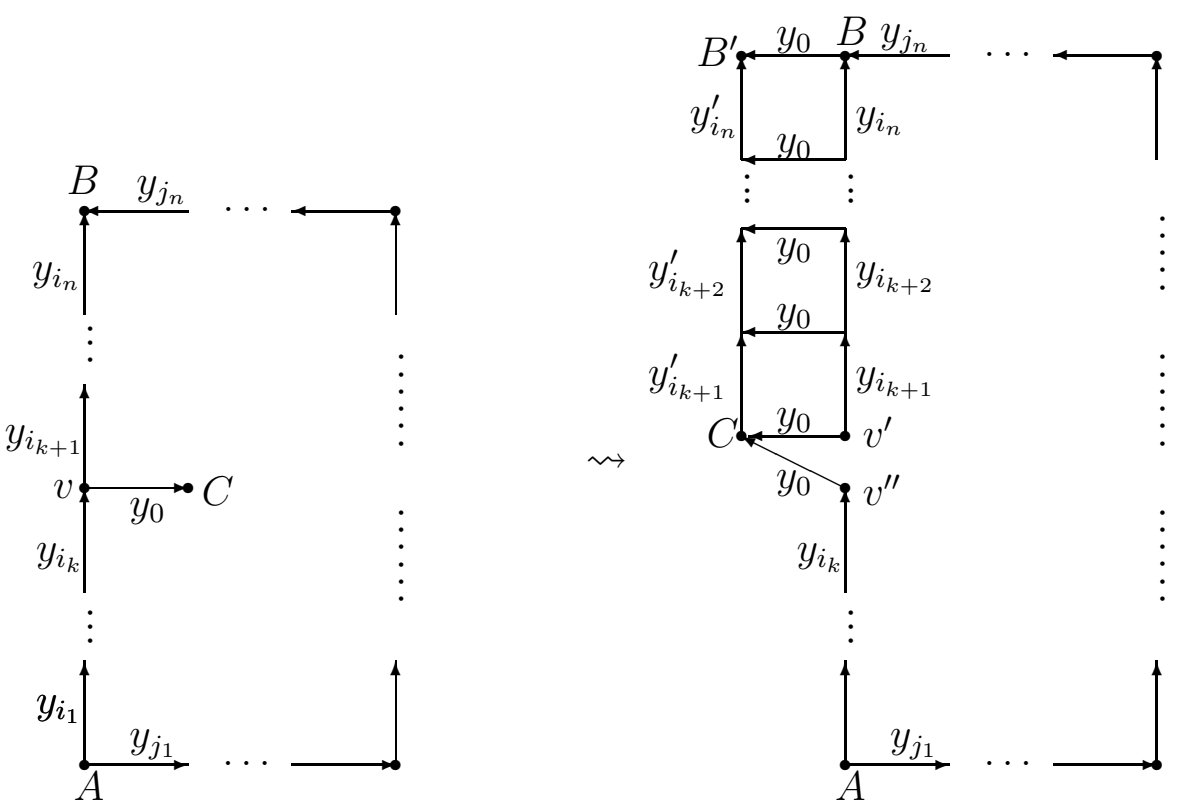

Fig.5

After gluing these quadrangles we obtain a new admissible disc diagram $D^{\prime}$ whose end is the vertex $B^{\prime}$ and the label of $\partial D^{\prime}$ is

$$
y_{i_{1}} \ldots y_{i_{k}} y_{0} y_{i_{k+1}}^{\prime} \ldots y_{i_{n}}^{\prime} y_{0}^{-1} y_{j_{n}}^{-1} \ldots y_{j_{1}}^{-1} \text {. }
$$


We call the transformation $D \rightsquigarrow D^{\prime}$ described above an admissible transformation III defined by the edge e.

Claim 11. If the elements $x_{i_{1}} \cdot \ldots \cdot x_{i_{k}} \cdot x_{0} \cdot x_{i_{k+1}}^{\prime} \cdot \ldots \cdot x_{i_{n}}^{\prime}$ and $x_{j_{1}} \cdot \ldots \cdot x_{j_{n}} \cdot x_{0}$, obtained from $x_{i_{1}} \cdot \ldots \cdot x_{i_{k}} \cdot x_{i_{k+1}} \cdot \ldots \cdot x_{i_{n}}$ and $x_{j_{1}} \cdot \ldots \cdot x_{j_{n}}$ after admissible transformation III defined by the edge $e$, are equivalent, then $x_{i_{1}} \cdot \ldots \cdot x_{i_{k}} \cdot x_{i_{k+1}} \cdot \ldots \cdot x_{i_{n}}$ and $x_{j_{1}} \cdot \ldots \cdot x_{j_{n}}$ are also equivalent.

Proof. Obvious.

Let $D$ be an admissible disc diagram such that its origin $A$ is a locally minimal vertex and its end $B$ is a locally maximal vertex. Let $C$ be a locally maximal vertex of $D, C \neq B$. Then $C \notin \partial D$, since there is only one locally maximal vertex belonging to $\partial D$, namely, the end $B$. We say that $C$ is visible if there is a vertex $v \in \partial D$, $v \neq A$, such that $v$ can be connected with $C$ by increasing path $l$ along edges of $D \backslash \partial D$ and such that $l \cap \partial D=v$. Let the path $l$ consist of edges $e_{1}, \ldots, e_{k}$ with labels $y_{1}, \ldots, y_{k}$. Perform the sequence of admissible transformations $I I I$ defined by the edges $e_{1}, \ldots, e_{k}$. As a result, we obtain a new admissible diagram $D^{\prime}$ in which the origin $A$ is a locally minimal vertex, the end $B^{\prime}$ is a locally maximal vertex, and the number of locally maximal vertices is strictly less that the number of locally maximal vertices of $D$. Such sequence of admissible transformations will be called a transformation decreasing the number of locally maximal vertices.

Let us return to the proof that $s_{1} \sim s_{2}$ if $\alpha_{G}\left(s_{1}\right)=\alpha_{G}\left(s_{2}\right)$, where $s_{1}=x_{i_{1}} \cdot \ldots \cdot x_{i_{n}}$ and $s_{2}=x_{j_{1}} \cdot \ldots \cdot x_{j_{n}}$ are two elements of the factorization semigroup $S(G, Y)$ over a $C$-group $(G, Y)$. Since $\alpha_{G}\left(s_{1}\right)=\alpha_{G}\left(s_{2}\right)$, the word $w=y_{i_{1}} \ldots y_{i_{n}} y_{j_{1}}^{-1} \ldots y_{j_{n}}^{-1}$ represents the unity of the group $G$. Therefore, by van Kampen Lemma, there is a plane disc diagram $D$ over the $C$-presentation of the group $G$ whose boundary label is the word $w$. By Lemmas 8 and 9, as it was mention above, we can assume that $D$ is a single disc. Conversely, an admissible disc diagram with boundary label $w=y_{i_{1}} \ldots y_{i_{n}} y_{j_{1}}^{-1} \ldots y_{j_{n}}^{-1}$ defines two elements $s_{1}(D)=x_{i_{1}} \cdot \ldots \cdot x_{i_{n}}$ and $s_{2}(D)=x_{j_{1}} \cdot \ldots \cdot x_{j_{n}}$ of $S(G, Y)$ such that $\alpha_{G}\left(s_{1}(D)\right)=\alpha_{G}\left(s_{2}(D)\right)$. Note that if $D \rightsquigarrow D^{\prime}$ is an admissible transformation $I$ or $I I$, or $I I I$, then, by Lemmas 8 , 9 and Claim 11, $s_{1}(D) \sim s_{2}(D)$ if and only if $s_{1}\left(D^{\prime}\right) \sim s_{2}\left(D^{\prime}\right)$. Therefore, without loss of generality, we can assume that $D$ is an admissible disc diagram such that

(i) the origin $A$ of $D$ is a locally minimal vertex,

(ii) the end $B$ of $D$ is a locally maximal vertex,

(iii) there is the only one visible locally maximal vertex of $D$, namely, the end $B$.

Let us show that if an admissible disc diagram $D$ satisfying conditions $(i)-($ iii $)$, then $s_{1}(D) \sim s_{2}(D)$. Indeed, if $D$ consists of a single quadrangle $Q$, then the origin $A$ is the bottom of $Q$ and the end $B$ is the top of $Q$. Obviously, in this case we have $s_{1}(D)=s_{2}(D)$ (see Fig. 1).

Now, let $D$ satisfy conditions $(i)-(i i i)$, have $K$ invisible locally maximal vertices and consist of $k$ quadrangles. Consider the edge $e_{1} \subset \partial_{l} D$ whose tail is $A$. Let $v_{1}$ be 
the head of $e_{1}$ and $Q$ a quadrangle such that $e_{1} \subset \partial Q$. Then the bottom of $Q$ is the origin $A$, since $A$ is a locally minimal vertex. Let $v_{2}$ be the top of $Q$ and $e_{2} \subset \partial Q$ the edge connecting $v_{1}$ and $v_{2}$.

There are two possibilities: either $e_{2} \subset \partial_{l} D$ and hence $v_{2} \in \partial_{l} D$, or $e_{2} \not \subset \partial_{l} D$. In the first case if $y_{i_{1}} y_{i_{2}} y_{l_{2}}^{-1} y_{l_{1}}^{-1}$ is the label of $\partial Q$, then $s_{1}(D)=x_{i_{1}} \cdot x_{i_{2}} \cdot\left(x_{i_{3}} \cdot \ldots\right.$. $\left.x_{i_{n}}\right)=x_{l_{1}} \cdot x_{l_{2}} \cdot\left(x_{i_{3}} \cdot \ldots \cdot x_{i_{n}}\right)$, and if we cut $Q$ from $D$, then we obtain a new admissible disc diagram $D^{\prime \prime}=D \backslash Q$ having only $k-1$ quadrangles and such that $s_{1}\left(D^{\prime \prime}\right)=x_{l_{1}} \cdot x_{l_{2}} \cdot\left(x_{i_{3}} \cdot \ldots \cdot x_{i_{n}}\right)$ and $s_{2}\left(D^{\prime \prime}\right)=x_{j_{1}} \cdot \ldots \cdot x_{j_{n}}$. Denote also $Q$ by $D^{\prime}$. Note that it is not obligatory that $D^{\prime \prime}$ satisfies conditions $(i)$ - (iii). Indeed, if the edge $e$ of $Q$ with label $x_{l_{1}}$ belongs to $\partial_{r} D$, then we must delete it to obtain admissible disc diagram. Therefore in this case the boundary label of $\partial D^{\prime \prime}$ is $y_{l_{2}}\left(y_{i_{3}} \ldots y_{i_{n}}\right) y_{j_{n}}^{-1} \ldots y_{j_{2}}^{-1}$ (since $y_{l_{1}}=y_{j_{1}}$ ) and if the origin of $D^{\prime \prime}$ is not a locally minimal vertex of $D^{\prime \prime}$, then we must perform a transformation $I$ which increases neither the number of locally maximal vertices nor the number of quadrangles. Next, it is possible that an invisible locally maximal vertex of $D$ becomes visible in $D^{\prime \prime}$. (It is possible only if $K>0$.) But, in this case we can perform an admissible transformation decreasing the number of locally maximal vertices and obtain a new admissible disc diagram having strictly less than $K$ invisible locally maximal vertices.

If $v_{2} \notin \partial D$, then there is an edge $e_{3}$ in $D$ whose tail is $v_{2}$, since, by assumption, $v_{2}$ is not a locally maximal vertex. Let $v_{3}$ is the head of $e_{3}$. If $v_{3} \notin \partial D$, then there is an edge $e_{4}$ whose tail is $v_{3}$, and so on. As a result, we can find an increasing path $l=\left(e_{2}, \ldots e_{m}\right)$ connecting the vertex $v_{1}$ and a vertex $v_{m}$ belonging to $\partial D$. There are two possibilities: either $v_{m} \in \partial_{l} D$ (see Fig. 6, Case $l$ ) or $v_{m} \in \partial_{r} D$ (see Fig. 6, Case $r$ ). In both cases the path $l$ divides $D$ into two disc diagrams $D^{\prime}$ and $D^{\prime \prime}$ each of which consists not more than $k-1$ quadrangles. If the label of $l$ is $y_{l_{2}} \ldots y_{l_{m}}$, then $s_{1}\left(D^{\prime}\right)=x_{i_{2}} \cdot \ldots \cdot x_{i_{m}}, s_{2}\left(D^{\prime}\right)=x_{l_{2}} \cdot \ldots \cdot x_{l_{m}}$ and $s_{1}\left(D^{\prime \prime}\right)=x_{i_{1}} \cdot x_{l_{2}} \cdot \ldots \cdot x_{l_{m}} \cdot x_{i_{m+1}} \cdot \ldots \cdot x_{i_{n}}, s_{2}\left(D^{\prime \prime}\right)=x_{j_{1}} \cdot \ldots \cdot x_{j_{n}}$ if $v_{2} \in \partial_{l} D$, and $s_{1}\left(D^{\prime}\right)=x_{i_{2}} \cdot \ldots \cdot x_{i_{n}}, s_{2}\left(D^{\prime}\right)=x_{l_{2}} \cdot \ldots \cdot x_{l_{m}} \cdot x_{j_{m+1}} \cdot \ldots \cdot x_{j_{n}}$ and $s_{1}\left(D^{\prime \prime}\right)=x_{i_{1}} \cdot x_{l_{2}} \cdot \ldots \cdot x_{l_{m}}$, $s_{2}\left(D^{\prime \prime}\right)=x_{j_{1}} \cdot \ldots \cdot x_{j_{m}}$ if $v_{2} \in \partial_{r} D$. If the end vertex $v_{m}$ of $D^{\prime}$ (resp., $D^{\prime \prime}$ ) in Case $l$ (resp., in Case $r$ ) is not locally maximal, then we perform an admissible transformation $I I$ of $D^{\prime}$ (resp., $D^{\prime \prime}$ ) which increases neither the number of quadrangles nor the number of locally maximal vertices. 


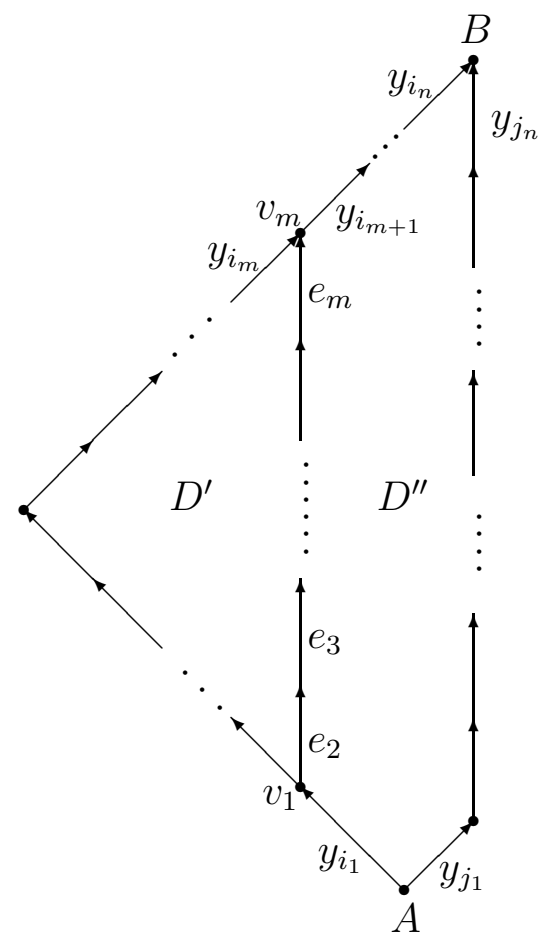

Case $l$

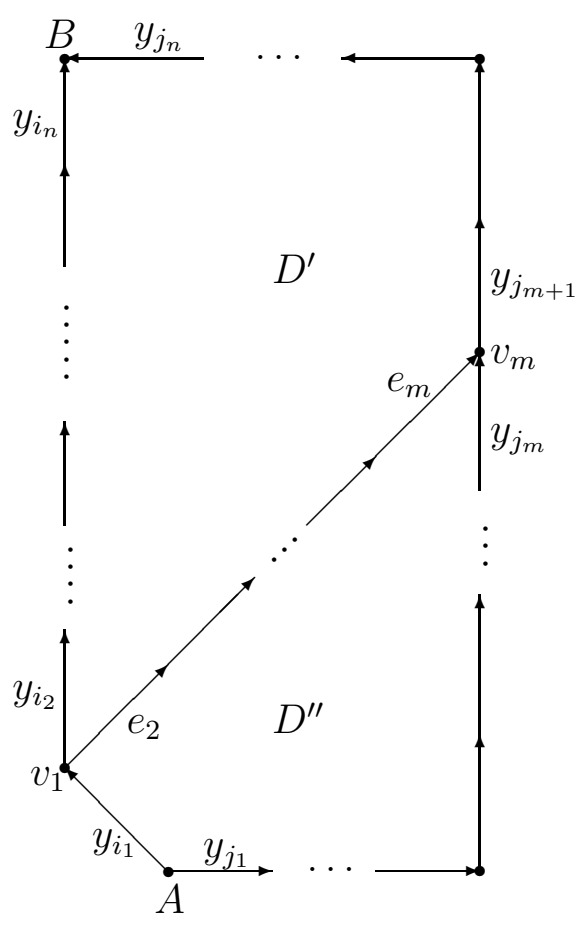

Case $r$

Fig. 6

To complete the proof of Theorem 2, we use two inductions: the first one on the number $K$ of the invisible locally maximal vertices of admissible disc diagrams satisfying conditions $(i)-($ iii $)$, and the second one on the number $k$ of quadrangles entering into diagrams. As it was shown above, in each step of inductions we can find a path $l$ dividing the admissible disc diagram $D$ satisfying conditions $(i)-($ iii) into two subdiagrams $D^{\prime}$ and $D^{\prime \prime}$ such that either (after an admissible transformation) $D^{\prime}$ and $D^{\prime \prime}$ have not more than $K$ invisible locally maximal vertices and have strictly less than $k$ quadrangles, or $D^{\prime}$ and $D^{\prime \prime}$ have strictly less than $K$ invisible locally maximal vertices (if $K>0$ ). By inductive assumptions, we have $s_{1}\left(D^{\prime}\right) \sim s_{2}\left(D^{\prime}\right)$ and $s_{1}\left(D^{\prime \prime}\right) \sim s_{2}\left(D^{\prime \prime}\right)$. Therefore, by Lemmas 8 and 9 , we have $s_{1}(D) \sim s_{2}(D)$.

3.2. Stability of the factorization semigroups over finite $C$-groups. Recall that the factorization semigroup $S(G, O)$ over an equipped group $(G, O)$ is called stable if there is an element $s \in S(G, O)$ such that $s_{1} \cdot s=s_{2} \cdot s$ for any two elements $s_{1}, s_{2} \in S(G, O)$ such that $\alpha_{G}\left(s_{1}\right)=\alpha_{G}\left(s_{2}\right)$ and $\tau\left(s_{1}\right)=\tau\left(s_{2}\right)$. 
Let $s_{1}, s_{2}$ be two equivalent elements of the factorization semigroup $S(G, Y)$ of a $C$-group $(G, Y)$. Denote by $e\left(s_{1}, s_{2}\right)$ the smallest number $k$ such that there is an element $s \in S(G, Y)$ of length $k$ and such that $s_{1} \cdot s=s_{2} \cdot s$.

As above, let $\Gamma=\Gamma_{1} \sqcup \cdots \sqcup \Gamma_{m}$ be the decomposition of the finite $C$-graph of a $C$-group $(G, Y)=G_{\Gamma}$ into the disjoint union of its connected components, $V_{i}=$ $\left\{v_{i, 1}, \ldots, v_{i, n_{i}}\right\}$ be the set of vertices of $\Gamma_{i}$, and $p_{i}$ be the period of vertices $v_{i, j}$. Any element $s \in S(G, Y)$ can be written in the form: $s=\prod_{v_{i, j} \in \Gamma} x_{v_{i, j}}^{k_{i, j}}$.

Consider a set

$$
R=\left\{s=\prod_{v_{i, j} \in \Gamma} x_{v_{i, j}}^{k_{i, j}} \in S(G, Y) \mid \sum_{j=1}^{n_{i}} k_{i, j} \leqslant p_{i} n_{i}, i=1, \ldots, m\right\},
$$

where $p_{i}$ are the periods of the $C$-generators $x_{v_{i, j}}$ of $G_{\Gamma}$ and let

$$
E=\left\{\left(s_{1}, s_{2}\right) \in R^{2} \mid s_{1} \sim s_{2}\right\} .
$$

Put

$$
e_{\Gamma}=\max _{\left(s_{1}, s_{2}\right) \in E} e\left(s_{1}, s_{2}\right)
$$

Lemma 10. We have $s_{1} \cdot s_{\Gamma}^{e_{\Gamma}}=s_{2} \cdot s_{\Gamma}^{e_{\Gamma}}$ for all $\left(s_{1}, s_{2}\right) \in E$, where $s_{\Gamma}$ is the canonical element of $S(G, Y)$.

Proof. For any $\left(s_{1}, s_{2}\right) \in E$ there is an element $s_{1,2}$ of $\ln \left(s_{1,2}\right) \leqslant e_{\Gamma}$ such that $s_{1} \cdot s_{1,2}=$ $s_{2} \cdot s_{1,2}$. By Lemma 2, there is an element $s_{1,2}^{\prime}$ such that $s_{1,2} \cdot s_{1,2}^{\prime}=s_{\Gamma}^{e_{\Gamma}}$. Therefore

$$
s_{1} \cdot s_{\Gamma}^{e_{\Gamma}}=s_{1} \cdot s_{1,2} \cdot s_{1,2}^{\prime}=s_{2} \cdot s_{1,2} \cdot s_{1,2}^{\prime}=s_{2} \cdot s_{\Gamma}^{e_{\Gamma}} .
$$

Theorem 3. Let $(G, Y)=G_{\Gamma}$ be a finite $C$-group. Then the semigroup $S(G, Y)$ is stable and $s_{\Gamma}^{e_{\Gamma}}$ is a stabilizing element.

Proof. Let $s_{1}, s_{2}$ be two elements of the factorization semigroup $S(G, Y)$ such that $\alpha_{G}\left(s_{1}\right)=\alpha_{G}\left(s_{2}\right)$ and $\tau\left(s_{1}\right)=\tau\left(s_{2}\right)$. Write them in the form

$$
s_{1}=\prod_{v_{i, j} \in \Gamma} x_{v_{i, j}}^{k_{i, j, 1}}, \quad s_{2}=\prod_{v_{i, j} \in \Gamma} x_{v_{i, j}}^{k_{i, j, 2}} .
$$

Since $\tau\left(s_{1}\right)=\tau\left(s_{2}\right)$, we have

$$
\sum_{j=1}^{n_{i}} k_{i, j, 1}=\sum_{j=1}^{n_{i}} k_{i, j, 2}, \quad i=1, \ldots, m .
$$

If $\sum_{j=1}^{n_{i}} k_{i, j, 1} \geqslant p_{i} n_{i}$ for some $i$, then there is $j_{1}$ (resp., $j_{2}$ ) such that $k_{i, j_{1}, 1} \geqslant p_{i}$ (resp., $k_{i, j_{2}, 2} \geqslant p_{i}$ ). If again

$$
k_{i, j_{1}, 1}-p_{i}+\sum_{j=1, j \neq j_{1}}^{n_{i}} k_{i, j, 1} \geqslant p_{i} n_{i}
$$


(resp.,

$$
\left.k_{i, j_{2}, 2}-p_{i}+\sum_{j=1, j \neq j_{2}}^{n_{i}} k_{i, j, 2} \geqslant p_{i} n_{i}\right),
$$

then either there is $j_{1}^{\prime}$ (resp., $j_{2}^{\prime}$ ) such that $k_{i, j_{1}^{\prime}, 1} \geqslant p_{i}$ (resp., $k_{i, j_{2}^{\prime}, 2} \geqslant p_{i}$ ) or $k_{i, j_{1}, 1}-p_{i} \geqslant$ $p_{i}$ (resp., $k_{i, j_{2}, 2}-p_{i} \geqslant p_{i}$ ). Continuing this process, as a result, we obtain that the elements $s_{1}$ and $s_{2}$ can be written in the following form:

$$
\begin{aligned}
& s_{1}=\prod_{i=1}^{m} \prod_{j=1}^{n_{i}} x_{v_{i, j}}^{p_{i} c_{i, j, 1}+r_{i, j, 1}}=\left(\prod_{i=1}^{m} \prod_{j=1}^{n_{i}} x_{v_{i, j}}^{p_{i} c_{i, j, 1}}\right) \cdot\left(\prod_{i=1}^{m} \prod_{j=1}^{n_{i}} x_{v_{i, j}}^{r_{i, j, 1}}\right), \\
& s_{2}=\prod_{i=1}^{m} \prod_{j=1}^{n_{i}} x_{v_{i, j}}^{p_{i} c_{i, j, 2}+r_{i, j, 2}}=\left(\prod_{i=1}^{m} \prod_{j=1}^{n_{i}} x_{v_{i, j}}^{p_{i} c_{i, j, 2}}\right) \cdot\left(\prod_{i=1}^{m} \prod_{j=1}^{n_{i}} x_{v_{i, j}}^{r_{i, j, 2}}\right),
\end{aligned}
$$

where

$$
\sum_{j=1}^{n_{i}} r_{i, j, 1}=\sum_{j=1}^{n_{i}} r_{i, j, 2}<n_{i} p_{i}, \quad i=1, \ldots, m
$$

and

$$
\sum_{j=1}^{n_{i}} c_{i, j, 1}=\sum_{j=1}^{n_{i}} c_{i, j, 2}, \quad i=1, \ldots, m .
$$

Since $\alpha_{G}\left(x_{v_{i, j_{1}}}^{p_{i}}\right)=\alpha_{G}\left(x_{v_{i, j_{2}}}^{p_{i}}\right)=y_{v_{i, 1}}^{p_{i}}$ for $1 \leqslant j_{1} \leqslant j_{2} \leqslant n_{i}$ and for all $i$ and since $\alpha_{G}\left(s_{1}\right)=\alpha_{G}\left(s_{2}\right)$, we have

$$
\alpha_{G}\left(\prod_{i=1}^{m} \prod_{j=1}^{n_{i}} x_{v_{i, j}}^{r_{i, j, 1}}\right)=\alpha_{G}\left(\prod_{i=1}^{m} \prod_{j=1}^{n_{i}} x_{v_{i, j}}^{r_{i, j, 2}}\right) .
$$

Then, by Theorem 2, we have

$$
\prod_{i=1}^{m} \prod_{j=1}^{n_{i}} x_{v_{i, j}}^{r_{i, j, 1}} \sim \prod_{i=1}^{m} \prod_{j=1}^{n_{i}} x_{v_{i, j}}^{r_{i, j, 2}}
$$

and by Lemma 10,

$$
\left(\prod_{i=1}^{m} \prod_{j=1}^{n_{i}} x_{v_{i, j}}^{r_{i, j, 1}}\right) \cdot s_{\Gamma}^{e_{\Gamma}}=\left(\prod_{i=1}^{m} \prod_{j=1}^{n_{i}} x_{v_{i, j}}^{r_{i, j, 2}}\right) \cdot s_{\Gamma}^{e_{\Gamma}} .
$$

Note that $s_{1} \cdot s_{\Gamma}^{e_{\Gamma}}$ (resp., $s_{2} \cdot s_{\Gamma}^{e_{\Gamma}}$ ) belongs to $S(G, Y)^{G}$. Therefore, by Lemma 1 ,

$$
s_{1} \cdot s_{\Gamma}^{e_{\Gamma}}=\left(\prod_{i=1}^{m} \prod_{j=1}^{n_{i}} x_{v_{i, j}}^{p_{i} c_{i, j, 1}}\right) \cdot\left(\prod_{i=1}^{m} \prod_{j=1}^{n_{i}} x_{v_{i, j}}^{r_{i, j, 1}}\right) \cdot s_{\Gamma}^{e_{\Gamma}}=\left(\prod_{i=1}^{m} x_{v_{i, 1}}^{p_{i} c_{i}}\right) \cdot\left(\prod_{i=1}^{m} \prod_{j=1}^{n_{i}} x_{v_{i, j}}^{r_{i, j, 1}}\right) \cdot s_{\Gamma}^{e_{\Gamma}}
$$

and

$$
s_{2} \cdot s_{\Gamma}^{e_{\Gamma}}=\left(\prod_{i=1}^{m} \prod_{j=1}^{n_{i}} x_{v_{i, j}}^{p_{i} c_{i, j, 2}}\right) \cdot\left(\prod_{i=1}^{m} \prod_{j=1}^{n_{i}} x_{v_{i, j}}^{r_{i, j, 2}}\right) \cdot s_{\Gamma}^{e_{\Gamma}}=\left(\prod_{i=1}^{m} x_{v_{i, 1}}^{p_{i} c_{i}}\right) \cdot\left(\prod_{i=1}^{m} \prod_{j=1}^{n_{i}} x_{v_{i, j}}^{r_{i, j, 2}}\right) \cdot s_{\Gamma}^{e_{\Gamma}},
$$


where $c_{i}=\sum_{j=1}^{n_{i}} c_{i, j, 1}=\sum_{j=1}^{n_{i}} c_{i, j, 2}$, and hence $s_{1} \cdot s_{\Gamma}^{e_{\Gamma}}=s_{2} \cdot s_{\Gamma}^{e_{\Gamma}}$.

Theorem 4. Let $(G, O)$ be an equipped finite group. The semigroup $S(G, O)$ (resp., $S(G, O)^{G}, S(G, O)_{1}$, and $\left.S(G, O)_{1}^{G}\right)$ over the group $G$ is stable if and only if the ambiguity index $a_{(G, O)}=1$.

Proof. The equipped group $(G, O)$ is equivalent to the finite $C$-group $\left(G_{\Gamma}, Y\right)$, where $\Gamma=\Gamma_{(G, O)}$, and there is an epimorphism $\beta_{(G, O)}: G_{\Gamma} \rightarrow G$, such that $H=\operatorname{ker} \beta \subset$ $Z\left(G_{\Gamma}\right)$. By Claim 9, the semigroups $S(G, O)$ and $S\left(G_{\Gamma}, Y\right)$ are naturally isomorphic. Let $\alpha_{G_{\Gamma}}$ and $\alpha_{G}$ be respectively the product homomorphisms of $S(G, O)$ to $G_{\Gamma}$ and $G$. Note that, by Theorem 3, for any positive integer $c$ the element $s_{\Gamma}^{c e_{\Gamma}}$ is a stabilizing element of $S\left(G_{\Gamma}, Y\right)$ over the group $G_{\Gamma}$ and there is a positive integer $c_{0}$ such that $\alpha_{G}\left(s_{\Gamma}^{c_{0} e_{\Gamma}}\right)=\mathbf{1} \in G$, since $G$ is a finite group.

If $a_{(G, O)}=\left|H \cap\left[G_{\Gamma}, G_{\Gamma}\right]\right|=1$, then for $s_{1}, s_{2} \in S(G, O)$ such that $\tau\left(s_{1}\right)=\tau\left(s_{2}\right)$, we have $\alpha_{G}\left(s_{1}\right)=\alpha_{G}\left(s_{2}\right)$ if and only if $\alpha_{G_{\Gamma}}\left(s_{1}\right)=\alpha_{G_{\Gamma}}\left(s_{2}\right)$. Therefore if $a_{(G, O)}=1$, then the semigroups $S(G, O), S(G, O)^{G}, S(G, O)_{1}$, and $S(G, O)_{1}^{G}$ over the group $G$ are stable and $s_{\Gamma}^{c_{0} e_{\Gamma}}$ is one of their stabilizing elements.

If $a_{(G, O)}>1$, then there is an element $g \in H \cap\left[G_{\Gamma}, G_{\Gamma}\right]$ such that $g \neq 1$. By Lemma 6, there are two elements $s_{1}, s_{2} \in S\left(G_{\Gamma}, Y\right)$ such that $g=\alpha_{G_{\Gamma}}\left(s_{1}\right) \alpha_{G_{\Gamma}}\left(s_{2}\right)^{-1}$. Therefore, $s_{1} \nsim s_{2}$, but $\alpha_{G}\left(s_{1}\right)=\alpha_{G}\left(s_{2}\right)$ and $\tau\left(s_{1}\right)=\tau\left(s_{2}\right)$, that is, the semigroups $S(G, O), S(G, O)^{G}, S(G, O)_{1}$, and $S(G, O)_{1}^{G}$ over the group $G$ are not stable (multiplying $s_{1}$ and $s_{2}$ by some element $s$, we can assume that $\left.s_{1}, s_{2} \in S(G, O)_{1}^{G}\right)$.

\section{UNIQUENESS OF FACTORIZATIONS IN THE CASE OF BIG ENOUGH NUMBER OF FACTORS}

4.1. The case of finite $C$-groups. Let $G_{\Gamma}=(G, Y)$ be a finite $C$-group and $m$ the number of connected components of the $C$-graph $\Gamma$.

Theorem 5. For each finite $C$-group $G_{\Gamma}=(G, Y)$, there is a constant $T \in \mathbb{N}$ such that if elements $s_{1}, s_{2} \in S(G, Y)^{G}$ satisfy the following conditions:

(i) $\tau_{i}\left(s_{1}\right) \geqslant T$ for $i=1, \ldots, m$;

(ii) $\alpha_{G}\left(s_{1}\right)=\alpha_{G}\left(s_{2}\right)$,

then $s_{1}=s_{2}$.

Proof. Put $\alpha=\alpha_{G}$ and note, first of all, that if $\alpha\left(s_{1}\right)=\alpha\left(s_{2}\right)$, then $\tau_{i}\left(s_{1}\right)=\tau_{i}\left(s_{2}\right)$ for $i=1, \ldots, m$, since $\tau_{i}\left(s_{j}\right)=a b_{i}\left(\alpha\left(s_{j}\right)\right)$.

Let us denote by $d_{\Gamma}$ the diameter of $\Gamma$ and, using the notations of section 3.2 , denote

$$
T_{1}=\left(d_{\Gamma}+1\right) \max _{1 \leqslant i \leqslant m} n_{i} p_{i}+1 .
$$


Let us show that any integer $T \geqslant T_{1}$ satisfies the conditions of Theorem 5. Indeed let $\tau_{i}\left(s_{1}\right) \geqslant T_{1}$ for $i=1, \ldots, m$. Then, by Corollary 1, the elements $s_{1}$ and $s_{2}$ can be written in the form: $s_{1}=s_{\Gamma}^{d_{\Gamma}} \cdot s_{1}^{\prime}$ and $s_{2}=s_{\Gamma}^{d_{\Gamma}} \cdot s_{2}^{\prime}$. We have

$$
\alpha\left(s_{1}^{\prime}\right)=\alpha\left(s_{1}\right) \alpha\left(s_{\Gamma}^{d_{\Gamma}}\right)^{-1}=\alpha\left(s_{2}\right) \alpha\left(s_{\Gamma}^{d_{\Gamma}}\right)^{-1}=\alpha\left(s_{2}^{\prime}\right)
$$

Therefore $s_{1}^{\prime} \sim s_{2}^{\prime}$ by Theorem 2 and hence $s_{1}=s_{2}$ by Theorem 3 ,

4.2. The case of equipped finite groups. Let $(G, O)$ be an equipped group equivalent to a finite $C$-group $G_{\Gamma}, \beta_{(G, O)}: G_{\Gamma} \rightarrow(G, O)$ the natural epimorphism of equipped groups, $H=\operatorname{ker} \beta_{(G, O)}, a_{(G, O)}=\left|H \cap\left[G_{\Gamma}, G_{\Gamma}\right]\right|$ the ambiguity index of $(G, O)$, and $m$ the number of connected components of $\Gamma$.

Theorem 6. For each equipped finite group $(G, O)$ there is a constant $T=T_{(G, O)}$ such that if for an element $s_{1} \in S(G, O)^{G}$ the ith type $\tau_{i}\left(s_{1}\right) \geqslant T$ for all $i=1, \ldots, m$, then there are $a_{(G, O)}$ elements $s_{1}, \ldots, s_{a_{(G, O)}} \in S(G, O)^{G}$ such that

(i) $s_{i} \neq s_{j}$ for $1 \leqslant i<j \leqslant a_{(G, O)}$;

(ii) $\tau\left(s_{i}\right)=\tau\left(s_{1}\right)$ for $1 \leqslant i \leqslant a_{(G, O)}$;

(iii) $\alpha_{G}\left(s_{i}\right)=\alpha_{G}\left(s_{1}\right)$ for $1 \leqslant i \leqslant a_{(G, O)}$;

(iv) if $s \in S(G, O)^{G}$ is such that $\tau(s)=\tau\left(s_{1}\right)$ and $\alpha_{G}(s)=\alpha_{G}\left(s_{1}\right)$, then $s=s_{i}$ for some $i, 1 \leqslant i \leqslant a_{(G, O)}$.

Proof. By Lemma 6, for $n \geqslant z_{H}$ each element $g_{i} \in H \cap\left[G_{\Gamma}, G_{\Gamma}\right]$, (where $g_{1}=\mathbf{1}$ ) can be presented in the form $g_{i}=\alpha_{G_{\Gamma}}\left(s_{i, n}\right) \alpha_{G_{\Gamma}}\left(s_{\Gamma}^{n}\right)^{-1}$, where $s_{i, n} \in S\left(G_{\Gamma}, Y\right)=S(G, O)$ (in particular, $s_{1, n}=s_{\Gamma}^{n}$ ). We have $s_{i, n} \neq s_{j, n}$ for $1 \leqslant i<j \leqslant a_{(G, O)}, \tau\left(s_{i, n}\right)=\tau\left(s_{1, n}\right)$ and $\alpha_{G}\left(s_{i, n}\right)=\alpha_{G}\left(s_{1, n}\right)$ for $1 \leqslant i \leqslant a_{(G, O)}$.

Put $T_{2}=\left(\max \left(d_{\Gamma}, z_{H}\right)+1\right) \max _{1 \leqslant i \leqslant m} n_{i} p_{i}+1$. By Corollary 1, if $T \geqslant T_{2}$, then the element $s_{1}$ can be written in the form: $s_{1}=s_{\Gamma}^{z_{H}} \cdot s_{1}^{\prime}$. Denote $s_{i}:=s_{i, z_{H}}^{z_{H}} \cdot s_{1}^{\prime}$. It is easy to see that the elements $s_{1}, \ldots, s_{a_{(G, O)}} \in S(G, O)^{G}$ satisfy conditions $(i)-(i i i)$ and if $s \in S(G, O)^{G}$ is such that $\tau(s)=\tau\left(s_{1}\right)$ and $\alpha_{G}(s)=\alpha_{G}\left(s_{1}\right)$, then $\alpha_{\Gamma}(s) \alpha_{G}\left(s_{1}\right)^{-1} \in$ $H \cap\left[G_{\Gamma}, G_{\Gamma}\right]$ and hence, by Theorem $\left[5, s=s_{i}\right.$ for some $i, 1 \leqslant i \leqslant a_{(G, O)}$.

Let $(G, O)$ be an equipped group, $O=C_{1} \sqcup \cdots \sqcup C_{m}$, where $C_{i}$ are conjugcy classes of $G$. Let for some $k<m$ the elements of the set $O^{\prime}=C_{1} \sqcup \cdots \sqcup C_{k}$ generate the group $G$. The embedding $i: O^{\prime} \hookrightarrow O$ defines subgraphs $\widetilde{\Gamma}^{\prime} \subset \Gamma^{\prime}=\Gamma_{1} \sqcup \cdots \sqcup \Gamma_{k}$ of the $C$-graph $\Gamma_{(G, O)}=\Gamma$, where $\widetilde{\Gamma}^{\prime}$ is the $C$-graph of the equipped group $\left(G, O^{\prime}\right)$. The subgraph $\Gamma^{\prime}$ is ample, since the the elements of $O^{\prime}$ generate the group $G$.

Let $G_{\Gamma}$ and $G_{\widetilde{\Gamma}^{\prime}}$ be $C$-groups equivalent respectively to $(G, O)$ and $\left(G, O^{\prime}\right)$, and $S\left(G_{\Gamma}, Y\right)$ and $S\left(G_{\widetilde{\Gamma}^{\prime}}, Y^{\prime}\right)$ their factorization semigroups. The embedding $i: O^{\prime} \hookrightarrow O$ defines a homomorphism $i_{*}: G_{\widetilde{\Gamma}^{\prime}} \rightarrow G_{\Gamma}$ of $C$-groups and an embedding

$$
i_{*}: S\left(G, O^{\prime}\right) \simeq S\left(G_{\widetilde{\Gamma}^{\prime}}, Y^{\prime}\right) \hookrightarrow S\left(G_{\Gamma}, Y\right) \simeq S(G, O)
$$

of semigroups such that $i_{*}\left(\alpha_{G_{\widetilde{\Gamma}^{\prime}}}(s)\right)=\alpha_{G_{\Gamma}}\left(i_{*}(s)\right)$ for all $s \in S\left(G_{\widetilde{\Gamma}^{\prime}}, Y^{\prime}\right)$. 
Theorem 7. Let $G$ be a finite group and $O^{\prime} \subset O$ be two its equipments such that the elements of $O^{\prime}$ generate the group $G$. Then, in notations used above, there is a constant $T=T_{\left(O, O^{\prime}\right)}$ such that if for an element $s_{1} \in S(G, O)^{G}$ the ith type $\tau_{i}\left(s_{1}\right) \geqslant$ $T$ for all $i=1, \ldots, k$, then there are not more than $a_{\left(G, O^{\prime}\right)}$ elements $s_{1}, \ldots, s_{n} \in$ $S(G, O)^{G}$ such that

(i) $s_{i} \neq s_{j}$ for $1 \leqslant i<j \leqslant n$;

(ii) $\tau\left(s_{i}\right)=\tau\left(s_{1}\right)$ for $1 \leqslant i \leqslant n$;

(iii) $\alpha_{G}\left(s_{i}\right)=\alpha_{G}\left(s_{1}\right)$ for $1 \leqslant i \leqslant n$,

where $a_{\left(G, O^{\prime}\right)}$ is the ambiguity index of $\left(G, O^{\prime}\right)$.

Proof. Let $T_{1}=2 d_{\Gamma^{\prime}} \max _{1 \leqslant i \leqslant k} n_{i} p_{i}$, where $d_{\Gamma^{\prime}}$ is the diameter of $\Gamma$ with respect to the ample subgraph $\Gamma^{\prime}, p_{i}$ and $n_{i}$, respectively, be the period and the number of vertices of the connected component $\Gamma_{i}$ of the graph $\Gamma$, and $T_{2}$ be a constant the existence of which for the equipped group $\left(G, O^{\prime}\right)$ is claimed in Theorem 6. Put $T=\max \left(T_{1}, T_{2}\right)$. By Proposition 4, if for $s \in S(G, Y)^{G}$ its $i$ th type $\tau_{i}(s) \geqslant T$ for all $i \leqslant k$, then $s$ can be written in the form: $s=\left(x_{v_{k+1,1}}^{a_{k+1}} \cdot \ldots \cdot x_{v_{m, 1}}^{a_{m}}\right) \cdot s_{\Gamma^{\prime}}^{d} \cdot s_{1}$, where $a_{i}=\tau_{i}(s)$ for $i=k+1, \ldots, m$ and $s_{1} \in S\left(G, O^{\prime}\right)$. Now, Theorem 7 follows from Theorem 6 applied to the element $s_{\Gamma^{\prime}}^{d} \cdot s_{1} \in S\left(G, O^{\prime}\right)^{G}$.

4.3. Generating functions. For each $\bar{k}=\left(k_{1}, \ldots, k_{m}\right) \in \mathbb{Z}_{\geqslant 0}^{m}$ denote by $h_{\bar{k}}$ the number of elements $s \in S(G, O)_{1}^{G}$ with $\tau(s)=\bar{k}$ and associate to $(G, O)$ a power series $\chi_{(G, O)}\left(t_{1}, \ldots, t_{m}\right):=\sum_{\bar{k} \in \mathbb{Z}_{\geqslant 0}^{m}} h_{\bar{k}} t_{1}^{k_{1}} \ldots t_{m}^{k_{m}}$.

Proposition 8. Let $G$ be a finite group and $C$ its conjugacy class such that the elements of $C$ generate the group $G$. Then $\chi_{(G, C)}(t)$ is a rational function.

Proof. Let $p$ be the period and $n$ the number of the vertices of $\Gamma_{(G, C)}$, and $a=a_{(G, C) \text { ) }}$ index of ambiguity of $(G, C)$. Consider a set of integers

$$
R=\left\{r \mid 0 \leqslant r \leqslant p n-1 \text { and } \exists i \equiv r(\bmod n p) \text { such that } h_{i}>0\right\}
$$

and let $i_{r}$ be the smallest $i$ for which $i \equiv r(\bmod n p)$ and $h_{i}>0$. Next, for each $r \in R$ choose a representative $s_{r} \in S(G, C)_{1}^{G}$ of length $\ln \left(s_{r}\right)=i_{r}$ and choose a constant $M$ such that $T=2 p n M>\max _{r \in R} i_{r}$ is a constant the existence of which is proved in Theorem 6.

Write $\chi_{(G, C)}(t)$ in the form: $\chi_{(G, C)}(t)=\chi_{<T}(t)+\chi_{\geqslant T}(t)$, where $\chi_{<T}(t)=\sum_{i=1}^{T-1} h_{i} t^{i}$. Note that $\chi_{<T}(t)$ is a polynomial. The function $\chi_{\geqslant T}(t)$ can be written in the form

$$
\chi_{\geqslant T}(t)=\sum_{r \in R} \sum_{j=0}^{\infty} h_{r+T+j p n} t^{r+T+j p n} .
$$


By Theorem 6, we have $h_{r+T+j p n}=a$ for each $r \in R$ and each $j \geqslant 0$, therefore

$$
\chi_{\geqslant T}(t)=a t^{T} \sum_{r \in R} t^{r} \sum_{j=0}^{\infty} t^{j p n}=\frac{a t^{T}}{1-t^{p n}} \sum_{r \in R} t^{r} .
$$

Example 2. If $G=\Sigma_{n}$ is the symmetric group and $O$ the set of transpositions, then by Clebsch - Hurwitz Theorem, $\chi_{(G, O)}=\frac{t^{2(n-1)}}{1-t^{2}}$.

A generalization of Proposition 8 is the following

Theorem 8. Let $G$ be a finite group and $O=C_{1} \sqcup \cdots \sqcup C_{m}$ a disjoint union of its conjugacy classes such that the elements of each class $C_{i}$ generate the group $G$. Then $\chi_{(G, O)}\left(t_{1}, \ldots, t_{m}\right)$ is a rational function.

Proof. It is similar to the proof of Proposition 8. We must note only that if $k_{i}$ is big enough, then it follows from Lemma 3 and Theorem 7 that

$$
0 \leqslant h_{\left(k_{1}, \ldots, k_{i-1}, k_{i}+p_{i} n_{i}, k_{i+1}, \ldots, k_{m}\right)} \leqslant h_{\left(k_{1}, \ldots, k_{i-1}, k_{i}, k_{i+1}, \ldots, k_{m}\right)} .
$$

Question 1. Is $\chi_{(G, O)}(\bar{t})$ a rational function for any equipped finite group $(G, O)$ ?

\section{Computation of the ambiguity index}

5.1. The word problem for finite $C$-groups. In this subsection we prove

Theorem 9. The finite $C$-groups have solvable word problem.

Proof. Let $\Gamma=\Gamma_{1} \sqcup \cdots \sqcup \Gamma_{m}$ be the $C$-graph of a finite $C$-group $\left(G_{\Gamma}, Y\right)$. By Claim 7, without loss of generality, we can assume that for each connected component $\Gamma_{i}$ of $\Gamma$ the period $p_{i}$ of its vertices is greater than one.

By Proposition 3, applying the relations of $C$-group given by $C$-graph $\Gamma$, any word in letters of $Y$ can be transformed into a word of the following form:

$$
w=c^{-1} y_{1,1}^{t_{1}} y_{2,1}^{t_{2}} \ldots y_{m, 1}^{t_{m}} \prod_{i=1}^{m} y_{i, 1}^{k_{i} p_{i}} y_{i, 1}^{a_{i, 1}} y_{i, 2}^{a_{i, 2}} \ldots y_{i, n_{i}}^{a_{i, n_{i}}},
$$

where $c$ is the canonical element of $G_{\Gamma}$ and the integers $k_{i}$ and $a_{i, j}$ satisfy the following relations and inequalities

$$
\begin{gathered}
\sum_{j=1}^{n_{i}} a_{i, j}+k_{i} p_{i}=n_{i} p_{i}, \\
0<a_{i, j} \leqslant p_{i}-1, \quad 0 \leqslant k_{i}<n_{i} .
\end{gathered}
$$

If two words $w_{1}$ and $w_{2}$ represent the same element $g \in G_{\Gamma}$, then $a b\left(w_{1}\right)=a b\left(w_{2}\right)=$ $\left(t_{1}, \ldots, t_{m}\right)$. Therefore, to prove Theorem, it suffices to show that there is a finite algorithm solving the following problem:

To recognize when two words

$$
w_{l}=\prod_{i=1}^{m} y_{i, 1}^{a_{i, 1, l}} y_{i, 2}^{a_{i, 2, l}} \ldots y_{i, n_{i}}^{a_{i, n_{i}, l}}, \quad l=1,2,
$$


satisfying the following relations and inequalities

$$
\begin{gathered}
\sum_{j=1}^{n_{i}} a_{i, j, l}=k_{i, l} p_{i}, \\
0 \leqslant a_{i, j, l} \leqslant p_{i}-1, \quad 0 \leqslant k_{i, l}<n_{i}
\end{gathered}
$$

represent the same element in $G_{\Gamma}$.

Note also that the words

$$
w_{l} \prod_{i=1}^{m} y_{i, 1}^{-k_{i, l} p_{i}}, \quad l=1,2,
$$

where $w_{l}$ are words from (16), represent elements of $\left[G_{\Gamma}, G_{\Gamma}\right]$.

In the sequel, to simplify notations, we will consider only the case when $m=1$, since the general case is similar. We will use the following notations: $\Gamma=\Gamma_{1}, n:=n_{1}$, $p:=p_{1},\left\{v_{1}, \ldots, v_{n}\right\}$ is the set of vertices of $\Gamma$ and $y_{i}$ is the $C$-generator of $G_{\Gamma}$ corresponding to a vertex $v_{i}$. Denote by $\mathcal{R}_{\Gamma}$ the set of relations in $G_{\Gamma}$ defined by the $C$ graph $\Gamma$ (see subsection 4). Remind that for each pair $\left(l_{1}, l_{2}\right)$ such that $1 \leqslant l_{1}, l_{2} \leqslant n$, there is unique $l_{3}$ (depending on $\left.\left(l_{1}, l_{2}\right)\right), 1 \leqslant l_{3} \leqslant n$, such that

$$
y_{l_{1}} y_{l_{2}} y_{l_{1}}^{-1} y_{l_{3}}^{-1} \in \mathcal{R}_{\Gamma} \text {. }
$$

Consider an equipped group $\left(\bar{G}=G_{\Gamma} / H, O\right)$, where $H$ is the subgroup of the center $Z\left(G_{\Gamma}\right)$ generated by the element $y_{1}^{p}=y_{i}^{p}, i=1, \ldots, n$. Denote by $f=f_{(G, O)}: G_{\Gamma} \rightarrow \bar{G}$ the natural epimorphism and by $f^{\prime}=f_{\left[G_{\Gamma}, G_{\Gamma}\right]}$ its restriction to $\left[G_{\Gamma}, G_{\Gamma}\right]$. The group $\bar{G}$ has the following presentation:

$$
\left.\bar{G}=\left\langle y_{1}, \ldots, y_{n}\right| R(\bar{y})=1 \text { for } R \in \mathcal{R}_{\Gamma} \text { and } y_{1}^{p}=\cdots=y_{n}^{p}=1\right\rangle .
$$

By Lemma 5, we have the commutative diagram

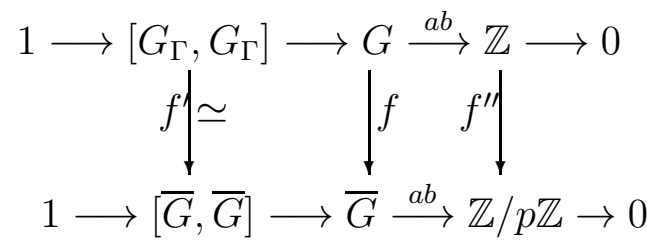

in which $f^{\prime}$ is an isomorphism, and $f$ and $f^{\prime \prime}$ are epimorphisms. It follows from the above considerations and diagram $(*)$ that each element $g \in \bar{G}$ can be represented by a word of the form:

$$
w=y_{1}^{a_{1}} y_{2}^{a_{2}} \ldots y_{n}^{a_{n}},
$$

where each integer $a_{j}$ satisfies the inequality

$$
0 \leqslant a_{j} \leqslant p-1
$$


and hence

$$
\sum_{j=1}^{n} a_{j} \leqslant n(p-1) .
$$

Denote by $W_{i}$ the set of positive words $w$ in letters of $Y$ whose lengths $\ln (w)=i$ (in particular, $W_{0}$ consists of the empty word), $W_{\leqslant k}=\bigcup_{i=0}^{k} W_{i}$, and by

$$
\widetilde{W}_{\leqslant k}=\left\{w \in W_{\leqslant k} \mid \text { each letter } y_{j} \in Y \text { enters in } w \text { less than } p \text { times }\right\} \text {. }
$$

Therefore, to prove Theorem 9, it suffices to show that there is a finite algorithm recognizing when two words $w_{1}, w_{2} \in \widetilde{W}_{\leqslant n(p-1)}$ represent the same element of $\bar{G}$. Prior to describe a such algorithm, we give two definitions. Let $w$ be a positive word in letters of $Y$ in which some letter $y_{j} \in Y$ enters sequentially $p$ times, that is, $w=w^{\prime} y_{j}^{p} w^{\prime \prime}$. Then we call the word $\bar{w}=w^{\prime} w^{\prime \prime}$ a reduction of $w$. For each subset $V \subset W_{\leqslant k}$, denote by $\bar{V}=\left\{\widetilde{w} \in \widetilde{W}_{\leqslant n(p-1)} \mid \exists w \in V\right.$ such that $\left.\widetilde{w}=\bar{w}\right\}$.

Let $\sigma_{1}, \ldots, \sigma_{k-1}$ be a set of Artin generators of the braid group $B_{k}$. Define an action of the group $B_{k}$ on the set $W_{i}$ as follows: if $i \neq k$, then this action is trivial; and if $i=k$, then for a word $w=y_{i_{1}} \ldots y_{i_{j-1}} y_{i_{j}} y_{i_{j+1}} y_{i_{j+2}} \ldots y_{i_{k}}$ its image $\sigma_{j}(w)=$ $y_{i_{1}} \ldots y_{i_{j-1}} y_{i^{\prime}} y_{i_{j}} y_{i_{j+2}} \ldots y_{i_{k}}$, where $i^{\prime}$ depends on the pair $\left(i_{j}, i_{j+1}\right)$ and it is defined by relation (20) if we put $l_{1}=i_{j}, l_{2}=i_{j+1}$, and $i^{\prime}=l_{3}$. Denote by

$$
B=B_{0} \times B_{1} \times \cdots \times B_{2 n(p-1)},
$$

where $B_{0}$ and $B_{1}$ are trivial groups. The action, defined above, makes it possible to define an action of $B$ on $W_{\leqslant 2 n(p-1)}=\bigcup_{i=0}^{2 n(p-1)} W_{i}$. For each subset $V$ of $W_{\leqslant 2 n(p-1)}$ denote by $B V$ the union of the orbits of the elements of $V$ under the action of $B$.

Let $N_{1}$ be the number of words in $\widetilde{W}_{\leqslant n(p-1)}$ and let us numerate them, $w_{1}, \ldots, w_{N_{1}}$, and consider each of them as a subset of $\widetilde{W}_{\leqslant n(p-1)}$ (denote by $\widetilde{V}_{j, 1}=\left\{w_{j}\right\}$ ). The algorithm can be described as follows. Let in the end of $(k-1)$ th step we have obtained a presentation of $\widetilde{W}_{\leqslant n(p-1)}$ as a disjoint union of its subsets,

$$
\widetilde{W}_{\leqslant n(p-1)}=\widetilde{V}_{1, k} \sqcup \cdots \sqcup \widetilde{V}_{N_{k}, k} \text {. }
$$

On the $k$ th step for each pair $(i, j) \in\left\{1, \ldots, N_{k}\right\}^{2}$, we form subsets

$$
\widetilde{V}_{i, k} \widetilde{V}_{j, k}=\left\{w=w^{\prime} w^{\prime \prime} \in W_{\leqslant 2 n(p-1)} \mid w^{\prime} \in \widetilde{V}_{i, k}, w^{\prime \prime} \in \widetilde{V}_{j, k}\right\} \subset W_{\leqslant 2 n(p-1)}
$$

and form the set of their orbits $\left\{B\left(\widetilde{V}_{i, k} \widetilde{V}_{j, k}\right)\right\}$. Define an equivalence relation induced by the following equivalence: two orbits $B\left(\widetilde{V}_{i_{1}, k} \widetilde{V}_{j, k}\right)$ and $B\left(\widetilde{V}_{i_{2}, k} \widetilde{V}_{j, k}\right)$ (resp., $B\left(\widetilde{V}_{i, k} \widetilde{V}_{j_{1}, k}\right)$ and $\left.B\left(\widetilde{V}_{i, k} \widetilde{V}_{j_{2}, k}\right)\right)$ are equivalent if there is $j_{0}$ (resp., $i_{0}$ ) such that

$$
B\left(\widetilde{V}_{i_{1}, k} \widetilde{V}_{j_{0}, k}\right) \cap B\left(\widetilde{V}_{i_{2}, k} \widetilde{V}_{j_{0}, k}\right) \neq \emptyset
$$

(resp., $B\left(\widetilde{V}_{i_{0}, k} \widetilde{V}_{j_{1}, k}\right) \cap B\left(\widetilde{V}_{i_{0}, k} \widetilde{V}_{j_{2}, k}\right) \neq \emptyset$ ) and for each equivalence class we unite the subsets $B\left(\widetilde{V}_{i, k} \widetilde{V}_{j, k}\right)$ belonging to this class. Denote the obtained subsets by $V_{1, k}, \ldots, V_{N_{k+1}, k}$ and put $\widetilde{V}_{i, k+1}:=\bar{V}_{i, k}$. 
It is easy to see that $N_{k+1} \leqslant N_{k}$ and $\widetilde{V}_{1, k+1} \sqcup \cdots \sqcup \widetilde{V}_{N_{k+1}, k+1}=\widetilde{W}_{\leqslant n(p-1)}$. The algorithm is stopped if $N_{k+1}=N_{k}$ and two words $w_{l}=y_{1}^{a_{1, l}} y_{2}^{a_{2, l}} \ldots y_{n}^{a_{n, l}}, l=1,2$, satisfying relation (17) and inequality (18) (in the case $m=1$ ) represent the same element in $G_{\Gamma}$ if and only if $w_{1}$ and $w_{2}$ belong to the same subset $\widetilde{V}_{i}:=\widetilde{V}_{i, k+1}$ for some $i$. To show this, let us introduce a group structure on the set $\widetilde{G}=\left\{\widetilde{V}_{i}\right\}$. By definition, the product of $\widetilde{V}_{i}$ and $\widetilde{V}_{j}$ is $\widetilde{V}_{i} \widetilde{V}_{j}=\overline{B\left(\widetilde{V}_{i} \widetilde{V}_{j}\right)}$. This product is well defined by construction of subsets $\widetilde{V}_{i}$ and since $N_{k+1}=N_{k}$. The unite in $\widetilde{G}$ is a subset $\widetilde{V}_{i_{0}}$ containing the empty word, the inverse element of $\widetilde{V}_{i}$ containing a word $w=y_{1}^{a_{1}} y_{2}^{a_{2}} \ldots y_{n}^{a_{n}}$ is the subset $\widetilde{V}_{j}$ containing the reduction of the word $w=y_{n}^{p-a_{n}} y_{n-1}^{p-a_{n-1}} \ldots y_{1}^{p-a_{1}}$. Let us renumber the subsets $\widetilde{V}_{j}$ so that for $i=1, \ldots, n$ the subset $\widetilde{V}_{i}$ contains the word $y_{i}$ and put $\widetilde{O}=\left\{\widetilde{V}_{1}, \ldots, \widetilde{V}_{n}\right\}$. Then it is easy to see that $\widetilde{O}$ is invariant under the inner automorphisms of $\widetilde{G}$ and the $C$-graph of the equipped group $(\widetilde{G}, \widetilde{O})$ coincides with $\Gamma$. Moreover, since to construct the elements of $\widetilde{G}$, we used only the relations from $\mathcal{R}_{\Gamma}$ and relations $y_{1}^{p}=\cdots=y_{n}^{p}=1$, there is an epimorphism $h:(\widetilde{G}, \widetilde{O}) \rightarrow(\bar{G}, \bar{O})$ such that the following diagram

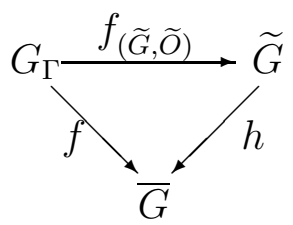

is commutative. Therefore $h([\widetilde{G}, \widetilde{G}])=[\bar{G}, \bar{G}]$ and since $f_{(\widetilde{G}, \widetilde{O})}\left(\left[G_{\Gamma}, G_{\Gamma}\right]\right)=[\widetilde{G}, \widetilde{G}]$ and $f^{\prime}:\left[G_{\Gamma}, G_{\Gamma}\right] \rightarrow[\bar{G}, \bar{G}]$ is an isomorphism, then $f_{(\widetilde{G}, \widetilde{O})}:\left[G_{\Gamma}, G_{\Gamma}\right] \rightarrow[\widetilde{G}, \widetilde{G}]$ is also an isomorphism.

5.2. Computation of the ambiguity index. We say that an equipped finite group $(G, O)$ is defined efficiently if there is a finite algorithm to enumerate the elements of $G$ (for example, if $G$ is given by its Cayley graph, or generators of $G$ as a subgroup of some symmetric group are given) and a representative of each class $C_{i} \subset O$ is also given.

Proposition 9. If an equipped finite group $(G, O)$ is defined efficiently, then there is a finite algorithm to compute the ambiguity index $a_{(G, O)}$.

Proof. Since $(G, O)$ is given efficiently, then, obviously, there is a finite algorithm to define completely the $C$-graph $\Gamma=\Gamma_{(G, O)}$.

Using the description of $\Gamma$, as in the proof of Theorem 9, we enumerate the subsets $\widetilde{V}_{i}$ of the set of words of the form (16) representing the same element in $G_{\Gamma}$ and which satisfy relations (17) and inequalities (18). After that for each $i$ we choose a word $w_{i} \in \widetilde{V}_{i}$ and check if $w_{i}$ represents the unity in $G$. The number of the sets $\widetilde{V}_{i}$ representing the unity of $G$ is equal to $a_{(G, O)}$. 


\section{REFERENCES}

[1] W. Fulton: Hurwitz schemes and irreducibility of moduli of algebraic curves. Ann. of Math., 90:3 (1969), $542-575$.

[2] A. Clebsch: Zür Theorie der Riemann'schen Fläche. Math. Ann., 6 (1872), 216 - 230.

[3] A. Hurwitz: Ueber Riemann'she Flächen mit gegebenen Verweigugspunkten. Math. Ann., 39, (1981), $1-61$.

[4] Vik.S. Kulikov: Factorization semigroups and irreducible components of Hurwitz space, will be published in Izv. Math. 75:4 (2011) (a primary version can be found in arXiv:1003.2953).

[5] Vik.S. Kulikov: Factorization semigroups and irreducible components of Hurwitz space. II, arXiv:1011.3619 (submitted to Izv. Math.).

[6] B. Wajnryb: Orbits of Hurwitz action for coverings of a sphere with two special fibres. Indag. Math. (N.S.), vol. 7 (1996), no. 4, $549-558$.

[7] Vik. S. Kulikov: Hurwitz curves. UMN, 2007, 62:6(378), 3 - 86.

[8] R.C. Lyndon and P.E. Schupp: Combinatorial group theory. Springer - verlag, Berlin Heidelberg - New York, 1977.

[9] A. Yu. Ol'shanskii: Geometry of defining relations in groups. "Mir", Moscow, 1980 (in Rassian).

Steklov Mathematical Institute

E-mail address: kulikov@mi.ras.ru 